\title{
Sirt6 protects cardiomyocytes against doxorubicin- induced cardiotoxicity by inhibiting P53/Fas- dependent cell death and augmenting endogenous antioxidant defense mechanisms
}

\section{Sisi Wu}

Core Facilities, West China Hospital, Sichuan University

\section{Xiaoxiao Wang}

Cancer Hospital, Chongqing University

\section{Mingming Tong}

Molecular Medicine Research Center, West China Hospital, Sichuan University

Li Fu

Core Facilities, West China Hospital, Sichuan University

\section{Yanjing Zhang}

Core Facilities, West China Hospital, Sichuan University

Jiayi Xu

Core Facilities, West China Hospital, Sichuan University

\section{Xuemei Chen}

Core Facilities, West China Hospital, Sichuan University

\section{Hongying Chen}

Core Facilities, West China Hospital, Sichuan University

Ruli Li

Molecular Medicine Research Center, West China Hospital, Sichuan University

Yao Wu

Molecular Medicine Research Center, West China Hospital, Sichuan University Juanjuan Xin

Molecular Medicine Research Center, West China Hospital, Sichuan University

Xin Yan

Molecular Medicine Research Center, West China Hospital, Sichuan University $\mathrm{He} \mathrm{Li}$

Molecular Medicine Research Center, West China Hospital, Sichuan University Jie Lan

Molecular Medicine Research Center, West China Hospital, Sichuan University Kunyue Xue

Molecular Medicine Research Center, West China Hospital, Sichuan University 


\section{Xue Li}

Molecular Medicine Research Center, West China Hospital, Sichuan University

\section{Caili Zhuo}

Molecular Medicine Research Center, West China Hospital, Sichuan University Jiang Wei ( $\nabla$ wcumsjw@scu.edu.cn )

Molecular Medicine Research Center, West China Hospital, Sichuan University

\section{Research Article}

Keywords: Doxorubicin, Cardiotoxicity, Sirt6, p53, Fas, FasL, Apoptosis, Necrosis

Posted Date: March 10th, 2021

DOI: https://doi.org/10.21203/rs.3.rs-263382/v1

License: (c) (i) This work is licensed under a Creative Commons Attribution 4.0 International License.

Read Full License 


\section{Abstract}

Sirt6, a class III NAD ${ }^{+}$-dependent deacetylase of the sirtuin family, is a highly specific $\mathrm{H} 3$ deacetylase and plays important roles in regulating cellular growth and death. The induction of oxidative stress and death are the crucial mechanisms involved in cardiomyocyte damage and cardiac dysfunction in doxorubicininduced cardiotoxocity, but the regulatory role of Sirt6 in the fate of DOX-impaired cardiomyocytes is poorly understood. In this study, we exposed heterozygous Sirt6 knockout $\left(\right.$ Sirt $\left.6^{+/-}\right)$mice and their littermates as well as cultured neonatal rat cardiomyocytes to DOX, then investigated how Sirt6 mitigates oxidative stress and myocardial injury in the DOX-treated myocardium. Sirt6 partial knockout or silencing worsened myocardial damage, cardiac remodeling, and oxidative stress in mice or cultured cardiomyocytes with DOX challenge. Cardiomyocytes infected with adenoviral constructs encoding Sirt6 showed reversal of this DOX-induced damage. Intriguingly, Sirt6 reduced oxidative stress injury by upregulating endogenous antioxidant levels, interacted with oxidative stress-stirred p53, and acted as a co-repressor of p53 in nuclei. Sirt6 was recruited by p53 to the promoter regions of the target genes Fas and FasL and further suppressed p53 transcription activity by reducing histone acetylation. Sirt6 inhibited Fas/FasL signaling and attenuated both Fas-FADD-caspase-8 apoptotic and Fas-RIP3 necrotic pathways. These results suggest that Sirt6 protects the heart against DOX-induced cardiotoxity by upregulating endogenous antioxidants, as well as suppressing oxidative stress and cell death signaling pathways dependent on ROS-stirred p53 transcriptional activation, thus reducing Fas-FasL-mediated apoptosis and necrosis.

\section{Introduction}

Doxorubicin (DOX) is a highly efficacious anticancer therapeutic drug but causes serious cardiotoxicity (Kalyanaraman et al. 2020; Zhang et al. 2009). Cardiomyocyte death due to apoptosis and necrosis is a critical mechanism of DOX-induced cardiotoxicity (Kalyanaraman et al. 2020; Zhang et al. 2009). Free radical-induced oxidative stresses play a pivotal role in DOX-induced cardiotoxicity, and other contributors including abnormalities of calcium handling, adrenergic impairment, and selective inhibition of cardiomyocyte-specific gene expression participate in its pathogenesis (Prathumsap et al. 2020; Kumari et al 2020). Most of the cardiomyocyte death can result from these cellular events (Zhang et al. 2009). Although DOX-induced cardiomyocyte death has been studied in depth for decades, the precise mechanisms are not completely elucidated (Prathumsap et al. 2020; Kumari et al 2020; Kalyanaraman et al. 2020; Zhang et al. 2009).

Sirtuins are an NAD-dependent deacetylase family that plays an essential role in delaying cell senescence and extending organismal lifespan by regulating diverse biological functions (Hall et al. 2013). The mammalian genome encodes seven sirtuin isoforms, Sirt1-Sirt7, with different intracellular distributions (Poulose et al. 2015). The isoforms are involved in a wide range of cellular processes from cell growth to longevity (Poulose et al. 2015; Hall et al. 2013). Sirt6, specifically located in the nucleus, is a stressresponsive protein deacetylase and mono-ADP ribosyltransferase enzyme that plays a leading role in regulating genomic stability, cell metabolism, inflammation, stress response and aging (Saiyang et al. 
2020; Poulose et al. 2015; Hall et al. 2013). Sirt6 has deacetylase activity toward histone H3K9Ac (acetylation on histone $\mathrm{H} 3$ lysine 9 ) and serves as a corepressor of the transcription factor hypoxia inducible factor $1 \mathrm{a}$, nuclear factor $\mathrm{KB}$ and c-Jun, to deacetylate histone H3K9Ac at their target promoters and downregulate the expression of a subset of their target genes (Saiyang et al. 2020; Poulose et al. 2015). Sirt6 protects cells against death by promoting resistance to DNA damage and oxidative stress (Saiyang et al. 2020; Beauharnois et al. 2013). Ran et al. observed that Sirt6 was recruited to the promoter of $\mathrm{BCl} 2$-associated $\mathrm{X}$, where it deacetylated $\mathrm{H} 3 \mathrm{~K} 9$ and suppressed its promoter activity to reduce $\mathrm{Bax}$ mRNA expression, then inhibited tumor cell apoptosis, thereby suggesting that Sirt6 can directly regulate cell death by interacting with apoptosis-related genes (Ran et al. 2016).

p53, a crucial tumor suppressor, is usually activated by oxidative stress inductions that are common responses to DOX exposure, and play a crucial role in DOX-induced toxicity (Shi et al. 2020; Liu et al. 2020; Gambino et al. 2013). p53 is also upregulated in cardiac tissues on DOX exposure (Men et al. 2020; Shizukuda et al. 2005). Once active p53 is bound to DNA, it can stimulate the transcription of many cell death genes (Hafner et al. 2019). p53-mediated signals play a significant role in DOX-induced cardiotoxicity (Men et al. 2020; Hafner et al. 2019; Zhu et al. 2009; Shizukuda et al. 2005). p53 inactivation protects hearts against DOX-induced damage and atrophy by inhibiting oxidative stress and disinhibiting mammalian target of rapamycin signaling (Zhu et al. 2009; Shizukuda et al. 2005). McSweeney et al. used RNA sequencing (McSweeney et al. 2019) to investigate DOX-induced transcriptomic changes in human induced pluripotent stem cell-derived cardiomyocytes and suggested that p53 is a key regulator of transcriptomic changes. In addition, the increased expression of death receptors (including Fas) and enriched exogenous apoptotic pathways were significantly associated with DOX-induced cardiac injury (McSweeney et al. 2019). Fas-mediated extrinsic apoptosis proceeds through the Fas-associated death domain (FADD)/caspase-8-dependent apoptotic pathway by binding to the receptor of its ligand, Fas ligand (Holler et al. 2000; Kaufmann et al. 2012). The FasL/Fas system also leads to necrosis by activating kinase receptor-interacting protein pathways (Holler et al. 2000; Zhang et al. 2010; Amir et al. 2009).

Some existing studies have suggested that there is a direct interaction between Sirt6 and p53 (Li et al. 2018; Ghosh et al. 2018). Sirt6 can serve as a co-activator of p53 (Li et al. 2018), and deacetylate p53 as a substrate (Ghosh et al. 2018). However, little is known about the role of Sirt6 in the regulation of cell death pathways (McSweeney et al. 2019; Hafner et al. 2019; Hall et al. 2013; Zhu et al. 2009). In this study, we investigated the role of Sirt6 on regulation of cardiomyocyte fate in DOX-insulted mice and cultured neonatal rat cardiomyocytes and identified its mechanism of action.

\section{Materials And Methods}

Reagents and antibodies

DOX, 5-bromo-2'-deoxyuridine (BrdUrd), lipid peroxidation (malondialdehyde, MDA) assay kit, catalase (CAT) and total superoxide dismutase (SOD) assay kits were from Sigma-Aldrich Chemicals (Men, USA). 
Protein A/G plus agarose was from Santa Cruz Biotechnology (Dallas, TX, USA). MitoSOX Red was from Invitrogen (Carlsbad, CA, USA). CardioTACS in situ apoptosis detection kit was from Trevigen (Gaithersburg, MD, USA). Lipofectamine 3000 and Trizol Reagent were from Invitrogen (Carlsbad, CA, USA). Annexin V-APC/7-AAD Cell Assay Kit was from BD Pharmingen (San Diego, CA, USA). Simple ChIP Enzymatic Chromatin IP Kit, and antibodies anti-total p53 (1:1000, \#2524), anti-acetyle-p53 (Lys382) (1:1000, \#2525), anti-phospho-p53 (Ser392) (1:1000, \#9281), anti-Sirt6 (1:1000, \#12486), anti-caspase-3 (1:1000, \#9662), anti-caspase-8 (1:1000, \#9746), anti-RIP1 (1:1000, \#3493), anti-RIP3 (1:1000, \#15828), anti-histone H3 (1:2000, 9715), anti-H3K9Ac (1:1000, \#9649), anti-Myc-Tag (9B11) (1:1000, \#2276), antiAcetylated-Lysine (1:100, \#9441), anti- $\beta$-actin (1:2000, \#4970) and the negative control for mouse Ig G (\#5415) were from Cell Signaling Technology (Danvers, MA, USA). The antibodies anti-Fas (1:1000, \#ab82419) and anti-FADD (1:1000, \#ab24533) were from Abcam (MA, USA). The antibodies anti-HMGB1 (1:1000, \#MAB16901-SP) and anti-FasL (1:1000, \#AB16982) were from R\&D Systems (Minneapolis, MN, USA) and Sigma-Aldrich Chemicals (St Louis, MO, USA), respectively. The membrane protein extraction kit was a Mem-PER ${ }^{\text {TM }}$ Plus Membrane Protein Extraction Reagent kit from Thermo Scientific (Catalog number: 89842, Rockford, IL, USA). The cytosol and nuclear protein extraction kit was a ReadyPrep ${ }^{\text {TM }}$ Protein Extraction Kit (cytoplasmic/nuclear) from Bio-Rad (cat\# 163-2089; Bio-Rad, Hercules, CA, USA). BCA protein assay kit was from Beyotime Institute of Biotechnology (Beijing, China). The PrimeScript RT reagent Kit with gDNA Eraser was from Takara Biotechnology (Dalian, China). All pairs of PCR primers were synthesized by the Chengdu Qingke Biotechnology (Chengdu, China). All other chemicals were of analytical grade.

Animals

Sirt6 $^{+/-}$(Sirt6 heterozygous) mice on a129Sv background were purchased from the Jackson laboratory (Bar Harbor, ME, USA). Sirt6 heterozygous mice $\left(\right.$ Sirt6 $\left.^{+/-}\right)$were backcrossed with wild-type (WT) C57BL/6J mice on a C57BL/6J background to obtain Sirt6 ${ }^{+/-}$mice and littermates (Wang et al. 2016). The mice were confirmed by PCR genotyping analysis (data not shown).

All procedures involving experimental animals were approved by the Animal Care and Use Committee of Sichuan University and performed in accordance with the Guidelines for Animal Experiments from the Committee of Medical Ethics, National Health Department of China.

In vivo DOX-induced cardiotoxicity mouse model

Male Sirt6 ${ }^{+/-}$and WT mice (8 to 12 weeks old) were treated with a 5-week protocol, which consisted of intraperitoneal injection (i.p.) of DOX at $8 \mathrm{mg} / \mathrm{kg}$ (body weight) or saline once a week for 4 weeks, and maintenance for another a week. All drug doses and injection regiments were based on previous reports with some modification (Wang et al. 2014).

Cardiac echocardiography and hemodynamics 
After the 5-week treatment, mice $(n=10)$ were anesthetized with isoflurane $(2 \%)$ in oxygen and positioned on a heated pad in a recumbent position (Wang et al. 2016; Wang et al. 2014). 2-D short-axis images were obtained by using a Vivid7 ultrasound imaging system (GE Health Medical, Milwaukee, WI, USA) operating at 12-MHz. Echocardiographic image acquisition and measurements were acquired from grayscale M-mode images at the mid-papillary level in the parasternal short-axis view, and 2-D mode images were obtained in the parasternal long- and short-axis views (Wang et al. 2016; Wang et al. 2014). After the echocardiography assessment, the mice were ventilated with a rodent ventilator (Harvard Apparatus, Holliston, MA, USA), then maintained with isoflurane ( $2 \%)$ in oxygen for anesthesia. An incision in the neck was made and a pressure-volume $(\mathrm{P}-\mathrm{V})$ catheter $1.2 \mathrm{~F}$ transducer $(4.5 \mathrm{~mm}$ electrode spacing, Serial No. 112B-B057, SClsense Inc., ON, Canada) was inserted into the left ventricle (LV) through the right carotid artery. After the P-V catheter was stabilized for several minutes, the signals were recorded by using an eight-channel physiological recorder (iWorx 308, iWorx/CB Sciences, Inc., Dover, NH, USA). The LV performance, including the LV peak systolic, end-diastolic pressures and heart rate were detected, while LV peak-negative developed pressure (dP/dtmin), LV pressure at peak positive developed pressure (dP/dtmax) was calculated (Wang et al. 2016; Wang et al. 2014). After hemodynamic measurements, mice were euthanized by pentobarbital overdose (i.p.), and hearts were removed and cut into three pieces. For histology, heart slices were fixed in $4 \%$ paraformaldehyde, paraffin-embedded, and cut into 4- $\mu \mathrm{m}$ serial sections for immunohistochemistry and the other two slices were snap-frozen in liquid nitrogen and stored at $-80^{\circ} \mathrm{C}$ (Wang et al. 2014).

Histology analysis

The histological sections were stained with hematoxylin-eosin (HE) solution, wheat germ agglutinin (WGA), and Masson trichrome and underwent TdT-mediated dUTP nick end-labeling (TUNEL) with standard protocols (Wang et al. 2016; Li et al. 2018). Cardiomyocyte size was measured from images of HE-stained sections and Alexa Fluor 488-conjugated WGA-stained sections and cardiomyocytes. About 100-200 random cardiomyocytes from each group $(n=10)$ were quantitatively assessed by using ImagePro Plus (Media Cybernetics, Silver Spring, MD, USA), and cardiomyocyte cross-sectional areas were visualized under a Zeiss microscope (AX10 imager A2 model, Germany) and presented in $\mu \mathrm{m}^{2}$ (Li et al. 2018). Myocardial fibrotic area was measured in five randomly selected fields per section on Masson's trichrome-stained sections from each group $(n=4)$ and the proportion of fibrosis were calculated by using Image-Pro Plus as the ratio of fibrotic area to total left ventricular (LV) area (Li et al. 2018; Ghosh et al. 2018; Wang et al. 2016; Zhang et al. 2010).

TUNEL staining used to assess DOX-induced cell apoptosis was analyzed by using a CardioTACS in situ apoptosis detection kit with the manufacturer's instructions (Wang et al. 2014). Five randomly areas from each slice were visualized for comparison of each experimental group. The apoptotic index (\%) was calculated as the ratio between TUNEL-positive cardiomyocyte nuclei and total number of nuclei $\times 100$ (Men et al. 2020; Wang et al. 2014).

Cardiomyocyte culture, RNA interference, adenoviral infection and treatment 
Neonatal rat cardiomyocytes were isolated from 0- to 2-day-old newborn Sprague-Dawley rats, which were sacrificed by decapitation, and cardiac ventricles were obtained from all rat pups for trypsin and collagenase II digestion as described (Wang et al. 2016; Li et al. 2018). After digestion, cells were suspended with Dulbecco's modified Eagle's medium (DMEM) with 10\% fetal bovine serum (FBS), 100 $\mathrm{U} / \mathrm{mL}$ penicillin and $100 \mathrm{U} / \mathrm{mL}$ streptomycin, then pre-plated for $1 \mathrm{~h}$ to remove any non-myocyte cells, then plated in cell culture dishes and placed overnight in incubator maintained at $5 \% \mathrm{CO}_{2}$ in air and $\nabla 95 \%$ humidity. On the following day, cells were cultured for $24 \mathrm{~h}$ in DMEM with $100 \mu \mathrm{M}$ BrdUrd to prevent nonmyocyte cell proliferation.

The rat Sirt6-siRNA (siRNA No., siG150505110012) and the control sequence (NTC, no target control) were from Guangzhou RiboBio Co., Ltd. (Guangzhou, China). Sirt6-siRNA and NTC were transfected into cells with Lipofectamine 3000 by the manufacturer's instructions (Wang et al. 2016). The replicationdefective adenoviral vectors expressing Sirt6 (Ad-Sirt6) and control green fluorescent protein (Ad-EGFP) was constructed by using the AdEasy 1 system instructions (Wang et al. 2014). In all siRNA-treated or adenovirus-treated experiments, cardiomyocytes were transfected with Sirt6 siRNA or control NTC siRNA oligonucleotides for $48 \mathrm{~h}$ or with Ad-Sirt6 or control Ad-EGFP adenovirus for $48 \mathrm{~h}$, then treated with DOX $(2 \mu \mathrm{M})$ for another $18 \mathrm{~h}$.

Malondiaedehyde (MDA) content and SOD2 and CAT enzyme activity assays

Heart tissues or cardiomyocytes were harvested and homogenized in $0.9 \%$ saline and centrifuged at $4^{\circ} \mathrm{C}$ for $15 \mathrm{~min}$ at 12, $000 \mathrm{rpm}$. The supernatant was collected. The protein concentration of lysates was measured with a BCA assay kit. Analysis of MDA content and both SOD2 and CAT activities in lysates of tissues and cardiomyocytes was according to the manufacturers' instructions (Wang et al. 2016; Wang et al. 2014).

RT-PCR assay

Total RNA was extracted by the phenol-chloroform extraction method with Trizol Reagent. A $1 \mu \mathrm{g}$ amount of total RNA was reverse-transcribed for cDNA synthesis with the PrimeScript RT reagent kit with gDNA Eraser. All relative mRNA levels were standardized to that of $\beta$-actin. The primer sequences are in supplemental Table 1 and RT-PCR procedures involved using a CFX96 Real-Time PCR Detection System (Bio-Rad Life Sciences).

Measurement of mitochondria reactive oxygen species production

Superoxide production was detected in living cardiomyocytes by using MitoSOX-red, a mitochondrionspecific hydroethidine-based fluorogenic dye following the manufacturer's instructions (Wang et al. 2016). Briefly, after treatment with siRNA or adenovirus, neonatal rat cardiomyocytes (NRCMs) were stained with MitoSOX-red reagent $(5 \mu \mathrm{M})$ with or without DOX $(2 \mu \mathrm{M})$ for $18 \mathrm{~h}$ in the dark at $37^{\circ} \mathrm{C}$, washed twice with phosphate buffered saline (PBS) and nuclei were stained with DAPI in anti-fade mounting 
buffer and visualized by Zeiss microscopy. Fluorescence intensity was analyzed by using Image $\mathrm{J}$ (Wang et al. 2016).

Apoptosis detected by flow cytometry

Primary cardiomyocytes $\left(5 \times 10^{5} / \mathrm{mL}\right)$ were cultured in six-well plates for $24 \mathrm{~h}$, then transfected with NTCsiRNA or Sirt6-siRNA for $48 \mathrm{~h}$ before incubation with or without DOX for $18 \mathrm{~h}$ or were overexpressed with Ad-EGFP or Ad-Sirt6 viruses and washed with PBS. Cells with no drugs were the control. Early/late apoptotic cells were measured by using the FACSAria SORP Cell Analyzer (BD, Biosciences, San Jose, CA, USA) with the Annexin V-APC/7-AAD Cell Assay Kit.

Western blot assay and immunoprecipitation

Western blot assay was used to determine protein expression as described (Wang et al. 2016; Li et al. 2018) including Sirt6, p53, caspase-8, caspase-3, RIP1, RIP3, Fas, FasL and FADD in cardiac tissues and cardiomyocytes. Total membrane proteins and cytosolic proteins were extracted to examine the expression of membrane Fas and FasL following the protein extraction kit as described (Hou et al. 2018). Nuclear proteins were extracted to detect the protein levels of high mobility group box 1 (HMGB1) and H3K9Ac by using a nuclear extraction kit (Wang et al. 2016). $\beta$-actin, Na/K-ATPase a1, and Histone H3 were used as a normalization control in whole cell homogenates, isolated membrane proteins and nuclei proteins, respectively.

The interacting partners of Sirt6 and p53 in myocardial cells were assessed by co-immunoprecipitation (Wang et al. 2016). Cardiomyocytes were harvested and suspended in $1 \mathrm{~mL}$ NP40 lysis buffer. Lysates were cleared by centrifugation at $13,000 \mathrm{rpm}$ for $10 \mathrm{~min}$. Protein $(500 \mu \mathrm{g})$ was immunoprecipitated with protein $\mathrm{A} / \mathrm{G}$ plus agarose, then incubated with $2.5 \mu \mathrm{g}$ anti-p53 antibody or non-immune mouse IgG control at $4{ }^{\circ} \mathrm{C}$ overnight, and immunoprecipitated proteins were detected by western blot methods as described (Wang et al. 2016).

Pull-down analysis

HEK293T cells were harvested at $48 \mathrm{~h}$ post-transfection with overexpression vectors of Flag-tagged Sirt6 (\#13817, Addgene) and Myc-p53 (\#MG50534, Sino Biological Inc. Beijing, China). Cell extracts were prepared, then pulled down with an anti-Flag antibody together with protein $A / G$ plus agarose. Immunoprecipitated proteins were analyzed by western blot assay with anti-Myc antibody as described previously (Amir et al. 2009)

Detection of acetyl-p53 level in nuclear protein

NRCMs at $5 \times 10^{6}$ cells $/ 10 \mathrm{~cm}$ plate were cultured for $24 \mathrm{~h}$, then transfected with NTC-siRNA or Sirt6-siRNA for $48 \mathrm{~h}$ before incubation with or without DOX for $18 \mathrm{~h}$, or NRCMs were overexpressed with Ad-EGFP or Ad-Sirt6 viruses before incubation with or without DOX for $18 \mathrm{~h}$. The nuclear proteins were extracted and immunoprecipitated with anti-acetylated-lysine antibody or non-immune mouse IgG control and protein 
A/G plus agarose. The amount of p53 in the nuclear extracts and immunoprecipitates were determined by immunoblotting with antibody p53 (Ghosh et al. 2018).

Chromatin immunoprecipitation (ChIP) assays

ChIP assay was performed as described previously by using the Simple ChIP Enzymatic Chromatin IP kit (Wang et al. 2016). Briefly, cells were cross-linked with $1 \%$ formaldehyde and digested with Micrococcal Nuclease into 150-900 bp DNA fragments, then a mouse anti-p53 antibody was added to the diluted soluble chromatin solution for $18 \mathrm{~h}$ at $4^{\circ} \mathrm{C}$ on a rotator, and a mouse IgG was used as a negative control; $1 \%$ of the total chromatin sample before immunoprecipitation (input). After being reverse crosslinked, eluted DNA was amplifed with specific Fas and FasL promoter primers (for a 174-bp fragment of Fas promoter: forward 5'-ATGAGTTGTGTGGGTGTCAG-3', reverse 5'-TAATGGAGGCAACAGAGCTAC-3'; for a 100 bp fragment of FasL promoter: forward 5'-CACTTACAGAACGGCACTAC-3' and reverse 5'ACTAGAGTCCACCAATCCCA-3'). The relative abundance of the studied DNA fragments in immunoprecipitated DNA was calculated with the ${ }^{\triangle \triangle} \mathrm{Ct}$ method.

\section{Statistical analysis}

The data are expressed as mean \pm SEM. Intergroup comparisons were analyzed by unpaired Student $t$ test and three or more groups were analyzed by one-way ANOVA followed by the Newman-Keuls tests. $P$ $<0.05$ was considered statistically significant.

\section{Results}

Sirt6 is downregulated by DOX insult

Cardiac Sirt6 level was significantly lower in Sirt6 ${ }^{+/-}$than WT mice, by $58.3 \%(P<0.01$, Fig. 1a). Five-week DOX challenge induced a significant decrease in the myocardial Sirt6 protein expression, with $39.5 \%$ and $74.8 \%$ less in WT and Sirt6 ${ }^{+/-}$than control hearts (both $P<0.01$; Fig. 1a). Sirt6 silence induced a significant decrease in Sirt6 level of neonatal rat cardiomyocytes by $72.5 \%$, compared with NTC-treated control cells $(P<0.01$, Fig. 1b). DOX treatment also significantly reduced Sirt6 expression, by $32.7 \%(P<$ $0.05)$ and $83.8 \%(P<0.01)$ in NTC-treated and Sirt6 siRNA-transfected neonatal rat cardiomyocytes, respectively (Fig. 1b). Ad-Sirt6 infection induced significant expression of Flag-Sirt6 in cardiomyocytes, which was significantly reduced by DOX insult (Supplementary Fig. 1).

Sirt6 deficiency aggravates body weight loss and promotes mortality in mice with DOX treatment

Mice receiving DOX showed rapid weight loss, which was deteriorated in Sirt6 ${ }^{+/-}$mice (Fig. 1C). However, the body weight for control WT and Sirt6 $6^{+/-}$mice gradually increased over 5 weeks, with no significant difference between the two groups. No control WT or Sirt $6^{+/-}$mice died. DOX exposure greatly decreased survival rate, with $46.5 \%$ and $76.3 \%$ WT and Sirt $6^{+/-}$mice dying 5 weeks after the first DOX injection (both 
$P<0.01$ vs controls, Fig. 1d). The survival rate was lower for DOX-treated Sirt6-deficient than DOX-treated WT mice $(P<0.05$, Fig. 1d).

Sirt6 deficiency aggravates cardiac atrophy after DOX treatment in vivo and ex vivo

DOX exposure induced left ventricular (LV) contractility decrease and myocardial structural changes (Wang et al. 2014). Five-week DOX treatment significant decreased ratio of LV weight to tibial length (LVW/TL) (Fig. 2a) and cross-sectional area of cardiomyocytes in WT mice (Fig. 2b, c) and increased interstitial fibrosis (Fig. 2b, d), for a distinct atrophic phenotype. However, the cardiac atrophy values were significantly lower and cardiac fibrosis was significantly higher for DOX-treated Sirt6 ${ }^{+/-}$mice than DOXtreated WT mice (Fig. 2b-d).

In cultured NRCMs, as compared with control NRCMs, DOX-treated NRCMs showed significantly decreased cross-sectional area (Fig. 2e), which was further significantly decreased with Sirt6 silencing but increased with Sirt6 overexpression (Fig. 2 f and Supplementary Fig. 1).

Sirt6 ablation increases DOX-induced apoptosis and necrosis in mouse hearts and primary cultured rat cardiomyocytes

Dox treatment significantly increased the number of TUNEL-stained cardiomyocytes in the myocardium of both WT and Sirt6 ${ }^{+/-}$mice; however, Sirt6 deficiency significantly increased the number of apoptotic nuclei in the Sirt6 ${ }^{+/-}$mouse myocardium as compared with WT mice (Fig. 3a). Sirt6 silencing significantly aggravated and Sirt6 overexpression significantly attenuated the apoptosis in response to DOX treatment (Fig. $3 \mathrm{~b}$ and c): the ratios of cleaved caspase-3 to pro-caspase-3 were higher, by $41.5 \%$, and lower, by $46.3 \%$, respectively, than in DOX-treated NTC-transfected cardiomyocytes. Flow cytometry assays exhibited that early apoptotic cardiomyocytes (Annexin- $V$ positive, $A P C^{+} 7-A^{-} D^{-}$, lower right quadrant) were significantly increased in number on DOX treatment (Fig. 3d, e). Sirt6 silencing significantly increased and Sirt6 overexpression reduced their number as compared with DOX treatment alone (Fig. 3d, e). Both Annexin-V and 7-AAD positive (APC+7-AAD', late apoptosis) and only 7-AAD positive cells (APC-7-AAD ${ }^{+}$, secondary necrosis) were significantly increased by DOX treatment (Fig. 3d, e; upper right quadrant). Sirt6 silencing significantly increased the number of late apoptotic and secondary necrotic cells, and Sirt6 overexpression reduced the number of both $A P C^{+} 7-A A D^{+}$and $A P C^{-} 7-A A D^{+}$cells as compared with DOX treatment alone (Fig. $3 \mathrm{~d}$ and e).

In addition, necrotic indicators, including loss of nuclear HMGB1, and release of myocardial enzymes, such as LDH, creatine kinase (CK) and CK-MB (CK-MB), were investigated. DOX treatment significantly decreased nuclear HMGB1 protein level in the myocardium of both WT and Sirt6 ${ }^{+/-}$mice, accompanied by a significant increase in plasma activity of LDH, CK and CK-MB (Fig. $3 f$ and Supplementary Fig. 2A). However, after DOX treatment, nuclear HMGB1 protein level was significantly lower, and activity of plasma myocardial enzymes was significantly higher in Sirt6 ${ }^{+/-}$than WT mice (Fig. $3 f$ and Supplementary Fig. 2A). DOX treatment significantly decreased HMGB1 protein levels in cardiomyocyte 
nuclei and increased the activity of myocardial enzymes in culture supernatants (Fig. $3 \mathrm{~g}$, $\mathrm{h}$ and Supplementary Fig. 2B, C). Sirt6 overexpression significantly attenuated and Sirt6 silencing aggravated the necrotic response to DOX treatment, with $43.2 \%$ higher $(P<0.01)$ and $37.3 \%$ lower $(P<0.05)$ HMGB1 protein level, respectively, in cardiomyocyte nuclei as compared with DOX-treated cardiomyocytes with null adenovirus infection or mock transfection (Fig. 3g, h). In addition, the activity of myocardial enzymes in culture supernatants was lower in DOX-treated NRCMs with Sirt6 overexpression and higher in DOXtreated NRCMs with Sirt6 silencing than with DOX treatment alone (Supplementary Fig. 2B, C).

Sirt6 deficiency accelerates LV dysfunction after DOX treatment

DOX-induced cardiac toxicity and atrophy are characterized by a decrease in both LV mass and cardiac functions (Zhang et al. 2009; Wang et al. 2014). DOX treatment significantly decreased

echocardiographic LV mass in Sirt6 ${ }^{+/-}$mice as compared with DOX-treated WT mice. Sirt6 deficiency also significantly impaired other parameters of cardiac remodeling, including LV posterior wall thickness at end-diastole and LV end-diastolic volume as well as the cardiac contractility indices derived from pressure volume, such as LV pressure, LV end-systolic pressure and $\pm \mathrm{dP} / \mathrm{dt}$ in DOX-treated Sirt6 ${ }^{+/-}$mice as compared with DOX-treated WT mice (Fig. 4 and Table 1). However, the LV preload index LV enddiastolic pressure was significantly greater in DOX-treated Sirt6 ${ }^{+/-}$than DOX-treated WT mice (Fig. 4 and Table 1). Consequently, cardiac function parameters, including stroke volume (SV), cardiac output (CO), fractional shortening (FS) and ejection fraction (EF) were significantly lower in DOX-treated Sirt6 ${ }^{+/-}$than DOX-treated WT mice (Fig. 4 and Table 1).

Sirt6 is necessary and sufficient to inhibit DOX-induced intracellular oxidative stress in vivo and ex vivo

DOX induces cardiac oxidative damage and dysfunction by inducing lipid peroxidation (indicated as MDA level) (Zhang et al. 2009; Prathumsap et al. 2020]. DOX treatment significantly increased MDA level in lysates of both cardiac tissues of WT mice and NRCMs. Sirt6 deficiency significantly augmented DOXinduced MDA levels in Sirt6 ${ }^{+/-}$mouse hearts and Sirt6-siRNA-transfected NRCMs over WT mice and NTC-treated cells with DOX treatment alone (Fig. 5a, b), although partial deletion of endogenous Sirt6 had no effect on baseline MDA content in Sirt6 ${ }^{+/-}$mouse hearts and Sirt6-silenced NRCMs (Fig. 5a, b). In addition, Sirt6 adenoviral overexpression significantly decreased MDA content in DOX-treated cardiomyocytes as compared with DOX-treated null-adenovirus-infected cells (Fig. 5c). MitoSOX Red, a mitochondrial ROS-sensitive indicator, was used to investigate mitochondrial ROS levels. Sirt6 gene silencing induced stronger mitochondria ROS staining in DOX-treated than non-treated cardiomyocytes (Fig. 5d), whereas Sirt6 overexpression conferred less mitochondrial ROS staining than in controls with DOX treatment alone (Fig. 5e).

We further observed that DOX treatment caused cardiac oxidative damage by downregulation of endogenous antioxidant enzymes. The mRNA levels of antioxidants, including Prx 5, CAT, SOD2 and glutathione peroxidase, and activity of $C A T$ and $S O D 2$ were significantly decreased in DOX-treated cardiac tissues and NRCMs (Fig. 5f-g and Supplementary Fig. 3A-C), parallel to increased ROS levels. Sirt6 gene 
silencing reduced the mRNA levels and activity of these endogenous antioxidases, and Sirt6 overexpression increased their mRNA levels and activity as compared with DOX alone-treated control counterparts (Fig. 5f-g and Supplementary Fig. 3B-C).

Sirt6-upregulated cardiomyocyte death is partially mediated by a p53-Fas-dependent pathway

The most important function of p53 is as a transcription factor (Hafner et al. 2019; Sullivan et al. 2018). We observed that DOX treatment significantly increased the protein levels of total p53, p53 phosphorylation at serine 392 (p53 Ser392-P) (Supplementary Fig. 4A, B), and pan-acetylated p53 (Fig. 6d, e) in NRCMs. We further investigated the potential interaction between Sirt6 and p53. Immunoprecipitates of endogenous p53 from NRCM lysates showed co-immunoprecipitation of endogenous Sirt 6 with p53, and this binding was further weakened by Sirt6 silencing but enhanced by Sirt6 overexpression (Fig. 6a, b). Also myc-tagged p53 and flag-tagged Sirt6 proteins overexpressed in HEK-293 cells co-immunoprecipitated (Fig. 6c).

Sirt6 silencing had no significant effect on pan-acetylated $p 53$ level, which was significantly reduced by Sirt6 overexpression (Fig. 6d, e). We further evaluated the mRNA levels of genes regulated by p53, including the negative regulators of the p53 pathway, Mdm2 and Cdkn2a, as well as the cellular death inducers, Bax, Bak, Fas, FasL, Noxa and Puma. DOX treatment significantly increased mRNA levels of p53, Bax, Bak, Fas, FasL, Noxa and Puma in cardiac tissues, and the transcription of these genes, as well as Mdm2, was significantly enhanced in DOX-treated NRCMs (Supplementary Fig. 5A-C). Only the mRNA levels of Fas, a death receptor, and its ligand, FasL, were regulated by Sirt6. Sirt6 knockout/silencing boosted mRNA levels of both FasL and Fas in DOX-treated cardiac tissues and NRCMs, which were blunted by Sirt6 overexpression (Supplementary Fig. 5A-C). However, the changes in Sirt6 levels did not affect the expression of the other five genes (Supplementary Fig. 5A-C). FasL exists in membrane-bound (mFasL) and soluble (sFasL) forms (Akiko et al. 2017). We confirmed that DOX treatment significantly increased both $\mathrm{mFasL}$ and Fas protein levels in the cellular membrane of NRCMs, which were significantly augmented by Sirt6 silencing but reduced by Sirt6 overexpression (Fig. 6f). In addition, sFasL protein change in culture supernatants of NRCMs was similar to that of mFasL (Fig. 6f). These results suggested that the p53-FasL-Fas pathway might mediate the regulation of Sirt6 on DOX-induced myocardial injury.

Sirt6 suppresses apoptosis dependent on caspase-8 and inhibits necrosis independent of RIP1 in cardiomyocytes

Fas-FasL interaction recruits FADD, and further recruits and activates caspase-8 to initiate apoptosis (Akiko et al. 2017; Kaufmann et al. 2012). DOX treatment significantly increased FADD and cleaved caspase-8 protein levels (Fig. 7a, b). RIP1 and RIP3 indeed play critical roles in programmed necrosis, and both of them are substrates of caspase-8 (Zhang et al. 2010; DeRoo et al. 2020). DOX treatment increased the inactive cleaved-RIP1 level significantly, which was parallel to caspase-8 activation. No RIP3 fragments were detected, and RIP3 protein level was significantly increased. Furthermore, Sirt6 silencing augmented the protein levels of FADD, activated caspase-8 fragment and RIP3, as well as the 
cleaved-RIP1 fragment (Fig. 7a), accompanied by a significant increase in apoptosis and necrosis in DOX-treated cardiomyocytes (Fig. 3a-h). The levels of all these proteins and fragments were significantly reduced with Sirt6 overexpression (Fig. 7b), parallel to a decrease in cardiomyocyte death (Fig. 3a-h).

Endogenous Sirt6 interacts with p53 for an important role in mediating H3K9 acetylation and the transcription expression of FasL and Fas in cardiomyocytes

Sirt6 usually acts as a co-repressor of transcription factors by modulating H3K9 acetylation (BoschPresegué et al. 2014). We used an antibody for p53 for ChIP assays to investigate the binding capacity of p53 to FasL and Fas promoters. p53 bound to both FasL and Fas promoters, and this binding was enhanced by DOX treatment alone (Fig. 8a, b). Sirt6 silencing significantly augmented and Sirt6 overexpression significantly reduced p53 binding capacity to both FasL and Fas promoters under DOX treatment, but Sirt6 silencing or overexpression had no apparent effect on control NRCMs (Fig. 8a, b). To reveal the role of Sirt6 in mediating the acetylation of histones, we further detected the acetylation level of H3K9. DOX treatment significantly increased nuclear H3K9 acetylation level, which was significantly augmented by Sirt6 silencing but reduced by Sirt6 overexpression (Fig. 8c, d).

\section{Discussion}

Cardiomyocyte apoptosis and necrosis could be a fundamental part of the myocardial process that initiates or aggravates heart failure (Prathumsap et al. 2020; Zhang et al. 2009). Although major efforts have been made to reveal the mechanism of anthracycline-induced cardiomyopathy, we still know very little about the molecular and cellular details (Kumari et al. 2020; Kalyanaraman et al. 2020; Zhang et al. 2010). Potential mechanisms include ROS generation, topoisomerase II inhibition, mitochondrial function degradation, proapoptotic pathway activation and $\mathrm{Ca}^{2+}$ mishandling (Shi et al. 2020; Prathumsap et al. 2020; Kalyanaraman et al. 2020; Zhang et al. 2009). However, ROS scavenging of iron chelators and the topoisomerase II- $\beta$ modulator dexrazoxane did not confer significant benefits, which indicates the presence of additional mechanisms (Kalyanaraman et al. 2020; Zhang et al. 2009). Whether Sirt6, a stress-responsive protein deacetylase and mono-ADP-ribosylation enzyme in various cardiac conditions, is involved in DOX-induced cardiotoxicity remained unclear (Saiyang et al. 2020; Poulose et al. 2015; Hall et al. 2013; Beauharnois et al. 2013). In the current study, we provide the first evidence that Sirt6 deficiency in vivo and in vitro aggravated DOX-induced cardiac atrophy, fibrosis, apoptosis and necrosis, thus exacerbating cardiac dysfunction and impairing mouse survival. Sirt6 overexpression in vitro mitigated DOX-induced cardiomyocyte injury. These data suggest that Sirt6-mediated inhibition of cell death is a major suppressor of DOX-induced cardiotoxicity. We demonstrate that Sirt6 can not only enhance the endogenous antioxidant defense but also interact with oxidative stress-induced p53. Sirt6 was recruited to the promoter regions of Fas/FasL, important death-inducing genes downstream of p53, and significantly decreased H3K9 acetylation levels as well as the binding of p53 to the Fas/FasL promoters, and then inhibited apoptosis/necrosis by suppressing the transcription of Fas/FasL genes (Fig. 8e). 
Previous studies indicate that cardiotoxicity is both an energy metabolism disturbance and oxidative stress injury after DOX chemotherapy, associated with metabolic disorders, mainly in lipids, amino acids, vitamins and energy metabolism (Prathumsap et al. 2020; Kumari et al. 2020; Kalyanaraman et al. 2020; Zhang et al. 2009). Sirt6 plays an essential role in glucose and lipid metabolism, inflammation, and genomic stability and is involved in the initiation and development of various cardiovascular diseases, including heart failure, cardiovascular remodeling, and atherosclerosis (Saiyang et al. 2020; Poulose et al. 2015; Hall et al. 2013; Beauharnois et al. 2013). Sirt6 can contribute to redox homeostasis by upregulating the antioxidant response (Saiyang et al. 2020; Beauharnois et al. 2013). In a previous study, we revealed that in ischemic hearts, an interaction between Sirt6 and Fox03a promoted the transcription and activity of the FoxO-dependent antioxidant enzyme genes SOD2 and CAT (Wang et al. 2016). In the present study, we confirmed that Sirt6 reduced DOX-induced excessive oxidative stress injury by upregulating endogenous antioxidants.

Accumulating evidence suggests that apoptosis and necrosis-induced cardiomyocyte death is an essential mechanism of DOX induced cardiotoxicity and cardiac dysfunction, so the mechanisms underlying the regulation of Sirt6 on cell fate have aroused our interest (Prathumsap et al. 2020; Zhang et al. 2009). Sirt6 is considered an important protective factor in cardiac ischemia/reperfusion injury, hypertrophy and atherosclerosis (Saiyang et al. 2020; Beauharnois et al. 2013); the downregulation of Sirt6 in DOX-treated hearts and cardiomyocytes may suggest a potential defect in the endogenous protective system. In addition, Sirts consume NAD ${ }^{+}$for exerting their enzymatic functions (Poulose et al. 2015; Hall et al. 2013). DOX treatment reduced the content of NAD + in cardiomyocytes, which might contribute to the decreased activity of Sirt6 (Gorini et al. 2018). Actually, the molecular mechanism mediating Sirt6 downregulation in DOX-treated hearts remains to be elucidated (Saiyang et al. 2020; Poulose et al. 2015; Hall et al. 2013; Beauharnois et al. 2013).

In hearts, p53 is a regulatory factor regulating cardiomyocyte cell cycle and fate (Men et al. 2020; Hafner et al. 2019). Basal p53 activity is essential in developing embryonic hearts and maintaining the normal myo-architecture of the heart and cardiac functions (Men et al. 2020). p53 expression and activity are upregulated in various myocardial diseases, including myocardial infarction, hypertrophic cardiomyopathy, atherosclerosis and chemotherapy-induced cardiotoxicity (Men et al. 2020). Oxidative stress is a determining factor of DOX cardiomyopathy and a potent inducer of p53 (Shi et al. 2020; Prathumsap et al. 2020; Liu et al. 2020; Gambino et al. 2013). Lots of evidence suggests ROS acts as a signaling molecule that triggers cascades including DNA damage response-independent JNK/p38MAPK and DNA damage response-dependent pathways to activate p53 (Shi et al. 2020; Liu et al. 2020; Gambino et al. 2013). We also confirmed that DOX induced a strong oxidative stress level and a significant increase in total, phosphorylated and acetylated p53 protein levels in cardiac tissues and cardiomyocytes, suggesting that DOX-induced excessive ROS might trigger the activation of p53 pathway. In fact, the role of p53 in anthracycline cardiotoxicity has been debated (Men et al. 2020; Li et al. 2019). Shizukuda et al. found that p53 gene disruption attenuated DOX-induced cardiotoxicity by inhibiting apoptosis and collapse of endogenous antioxidant enzymes (Shizukuda et al. 2005). However, Li et al. reveal a non- 
canonical tumor suppressor activity of p53 in that retained mitochondrial regulation prevented low-dose DOX-induced cardiac dysfunction in a mouse model (Li et al. 2019). Thus, p53 may involve a complex mechanism for controlling cell fate (Li et al. 2019; Antonina et al. 2019). We observed that as a protein deacetylase, Sirt6 overexpression reduced pan-acetylated p53 level, which is comparable to results reported by Ghosh et al. (Ghosh et al. 2018), finding that Sirt6 directly deacetylated p53 at lysine 381 but not K382 to negatively regulate the stability and activity of p53. Our results suggest that Sirt6 might directly suppress DOX-stirred p53 activity by reducing p53 stability via its deacetylation on p53.

p53 responds to various forms of cell stress as a DNA sequence-specific transcriptional regulator, which controls the expression of many genes involved in cell outcomes, including cell cycle arrest and cell death(Antonina et al. 2019; Laptenko et al. 2006). p53 interactions multiply with coactivators, coinhibitors, and components of general transcriptional machinery, which allows p53 to promote or suppress the transcription of different target genes (Antonina et al. 2019; Laptenko et al. 2006). We confirm the direct physiological binding and interaction between Sirt6 and p53 in cardiomyocytes, so Sirt6 may be a potential candidate co-factor for $\mathrm{p} 53$. This finding is comparable to those reported by Li et al. (Li et al. 2018), showing that Sirt6 co-operated with p53 as a co-activator.

Fas and FasL are involved in the regulation of cell death (Yamada et al. 2017; Kaufmann et al. 2012). We observed that only the transcription of Fas and its legend FasL were regulated by Sirt6 silencing and overexpression. These results indicate that p53 downstream targets Fas and FasL are involved in the regulation of Sirt6 on DOX-induced cardiomyocyte death. We further investigated the regulation of Sirt6 in the interaction between p53 and Fas and FasL promoters by ChIP assay. The recruitment of p53 to the Fas and FasL promoters was further enhanced by Sirt6 silencing, accompanied by increased H3K9 acetylation; these bindings were impaired by Sirt6 overexpression, which was consistent with decreased $\mathrm{H} 3 \mathrm{~K} 9$ acetylation. Acetylation of histones can increase the expression of genes via transcription activation (Yang et al. 2020). Deacetylase-induced deacetylation has the opposite effect, inducing decreased gene expression (Yang et al. 2020; Tao et al. 2014). Thus, Sirt6 may be a co-repressor of p53, recruited to promoters of Fas and FasL, and further suppresses p53 transcription activity by reducing histone acetylation.

Cardiomyocytes express both Fas and FasL (Stephanou et al. 2001). When bound by FasL, Fas initiates signaling through their cytoplasmic death domains to induce both apoptotic and necrotic cell death pathways (Kaufmann et al. 2012; Kavurma et al. 2003). FADD, an essential adaptor protein in cell death, binds to the death domain of Fas via its own death domain and recruits procaspase-8 via the interaction of death effector domains, then a death-inducing signaling complex is formed to induce procaspase-8 being activated by self-cleavage (Tourneur et al. 2010). Active caspase-8 induces apoptosis by directly activating downstream effector caspases, including caspase-3 (Kaufmann et al. 2012; Tourneur et al. 2010). We confirmed that activated caspase-8 induced caspase-3 cleavage after activation of the FasFADD pathway in DOX-treated cardiomyocytes, which suggests that DOX induced apoptosis depends on Fas-FADD-caspase-8. These results were comparable to those by Ueno et al. (Ueno et al. 2006), showing increased FasL mRNA expression, caspase-8 activity and apoptosis in hearts of DOX-treated rats. 
In addition to apoptosis, necrosis is an important type of programmed cell death (Antonina et al. 2019; Zhang et al. 2009). RIP1 and RIP3, the important serine-threonine kinases, have been identified as essential regulators of caspase-independent necrosis (DeRoo et al. 2020; Zhang et al. 2016). In general, in response to the activated signal of Fas, RIP1 binds to Fas with its death domain and acts as a multifunctional adaptor protein, then the C-terminal RIP homotypic interaction motif domain of RIP1 allows it to combine with and phosphorylate RIP3, to form an activated RIP1/RIP3 necrosome (Zhang et al. 2016; Kaufmann et al. 2012). The necrosome then induces mixed-lineage kinase domain-like protein (MLKL) to form a large MLKL octamer that serves as the effector for cell death [20]. However, Zhang et al. revealed another mechanism in cardiomyocyte necrosis induced by DOX, that is, RIP3 mediates myocardial necroptosis via CaMKII activation rather than RIP1 and MLKL (Zhang et al. 2016). Our results were comparable to Zhang et al. (Zhang et al. 2016), confirming that DOX induced RIP3-dependent necrosis in cardiomyocytes, but RIP1 might be cleaved by activated caspase-8.

In conclusion, as summarized in Fig. 8e, Sirt6 plays an essential role in preserving the homeostasis of redox and antioxidants as well as the survival and death of cardiomyocytes. It shows strong protection against oxidative stress and cell death in DOX-treated cardiomyocytes. Sirt6 reduces oxidative stress injury by up-regulating endogenous antioxidants, and interacts with ROS-actived p53 and serves as a corepressor of p53. It is recruited to the gene promoters of death receptor, Fas, and its ligand, FasL, and further suppresses p53 transcription activity by reducing histone acetylation. The inhibition of Sirt6 on death receptor signaling leads to impaired Fas-FADD-caspase-8 apoptotic and Fas-RIP3 necrotic pathways. These findings have far-reaching implications for treating chemotherapy-induced cardiotoxicity and for various other diseases characterized by the activation of death receptor signaling.

\section{Abbreviations}

CK creatine kinase

CK-MB creatine kinase-MB

CO cardiac output

DMSO dimethyl sulfoxide

DOX doxorubicin

$\mathrm{dP}_{\max } \quad$ maximal value of the first derivative of $L V$ pressure

$\mathrm{dP}_{\min } \quad$ minimal value of the first derivative of LV pressure

EDV end-diastolic volume

EF ejection fraction 
ESV end-systolic volume

FADD Fas associated via death domain

Fas tumor necrosis factor receptor superfamily member 6

FasL Fas receptor-Fas ligand

FS fractional shortening

GFP green fluorescent protein

Gpx1 glutathione peroxidase 1

H3K9Ac acetylation on histone H3 lysine 9

HMGB1 high mobility group box 1

KO knockout

LDH lactate dehydrogenase

LV left ventricular

LVEDD LV end diastolic diameter

LVEDV LV end-diastolic volume

LVEDP LV end-diastolic pressure

LVESP LV end-systolic pressure

LVIDs LV internal dimension in systole

LVIDd LV internal dimension in diastole

LVP LV pressure

LVPWd LV posterior wall thickness at end-diastole

LVSd left ventricle systolic diameter

LVW/TL left heart ventricle weight/tibia length

NRCMs neonatal rat cardiomyocytes

Prx5 Peroxiredoxin 5 
ROS reactive oxygen species

SOD2 Mn-dependent superoxide dismutase 2

SV stroke volume

WT wild type

\section{Declarations}

\section{Authors' contribution}

SSW: investigation, experiments, data collection, writing-original draft. XXW, MMT: idea, formal analysis. LF, YJZ, JYX, XMC, HYC: Validation, formal analysis. XY, RLL, YW, JJX, HL, LJ, XL: animal experiment. KYX, CLZ: provision of materials and instrumentation. WJ: funding acquisition, writingreviewing and editing.

\section{Acknowledgements}

This work was supported by the National Natural Science Foundation of China (grant nos. 81870221, 81670249, 82070299, 31271226 and 31071001 to Dr. Wei Jiang, and 81900276 to Dr. Ruli Li). Special thanks to Laura Smales for help in editing and revising the manuscript.

\section{Code availability}

Not applicable.

\section{Funding}

This work was supported by the National Natural Science Foundation of China (grant nos. 81870221, 81670249, 82070299, 31271226 and 31071001 to Dr. Wei Jiang, and 81900276 to Dr. Ruli Li).

\section{Availability of data and materials}

The authors declare that all materials and data generated or analyzed in this study are available within this article and the supplementary materials.

\section{Ethics approval and consent to participate}

N/A. There were no any human experiments in this study.

\section{Consent for publication}

All authors agreed to submit the manuscript for publication in Cell Biology and Toxicology.

\section{Competing interests}


All authors declare that they have no competing interests.

\section{References}

Amir S, Wang R, Simons JW, Mabjeesh NJ. SEPT9_v1 up-regulates hypoxia-inducible factor 1 by preventing its RACK1-mediated degradation. J Biol Chem. 2009; 284(17):11142-51. https://doi.org/10.1074/jbc.M808348200.

Beauharnois JM, Bolívar BE, Welch JT. Sirtuin 6: a review of biological effects and potential therapeutic properties. Mol Biosyst. 2013; 9(7):1789-806. https://doi.org/10.1039/c3mb00001j.

Bosch-Presegué L, Vaquero A. Sirtuins in stress response: guardians of the genome. Oncogene. 2014; 33(29):3764-75. https://doi.org/10.1038/onc.2013.344.

DeRoo E, Zhou T, Liu B. The Role of RIPK1 and RIPK3 in Cardiovascular Disease. Int J Mol Sci. 2020; 21(21):8174. https://doi.org/10.3390/ijms21218174

Gambino V, De Michele G, Venezia O, Migliaccio P, Dall'Olio V, Bernard L, et al. Oxidative stress activates a specific p53 transcriptional response that regulates cellular senescence and aging. Aging Cell. 2013; 12(3):435-45. https://doi.org/10.1111/acel.12060.

Ghosh S, Wong SK, Jiang Z, Liu B, Wang Y, Hao Q, et al. Haploinsufficiency of Trp53 dramatically extends the lifespan of Sirt6-deficient mice. Elife. 2018; 7:e32127. https://doi.org/10.7554/eLife.32127.

Gorini S, De Angelis A, Berrino L, Malara N, Rosano G, Ferraro E. Chemotherapeutic Drugs and Mitochondrial Dysfunction: Focus on Doxorubicin, Trastuzumab, and Sunitinib. Oxid Med Cell Longev. 2018; 2018:7582730. https://doi.org/10.1155/2018/7582730.

Hafner A, Bulyk ML, Jambhekar A, Lahav G. The multiple mechanisms that regulate p53 activity and cell fate. Nat Rev Mol Cell Biol. 2019; 20(4):199-210. https://doi.org/10.1038/s41580-019-0110-x.

Hall JA, Dominy JE, Lee Y, Puigserver P. The sirtuin family's role in aging and age-associated pathologies. J Clin Invest. 2013; 123(3):973-9. https://doi.org/10.1172/JCI64094.

Holler N, Zaru R, Micheau O, Thome M, Attinger A, Valitutti S, et al. Fas triggers an alternative, caspase-8independent cell death pathway using the kinase RIP as effector molecule. Nat Immunol. 2000; 1(6): 48995. https://doi.org/10.1038/82732.

Hou L, Wang K, Zhang C, Sun F, Che Y, Zhao X, et al. Complement receptor 3 mediates NADPH oxidase activation and dopaminergic neurodegeneration through a Src-Erk-dependent pathway. Redox Biol. 2018; 14:250-60. https://doi.org/10.1016/j.redox.2017.09.017.

Kalyanaraman B. Teaching the basics of the mechanism of doxorubicin-induced cardiotoxicity: Have we been barking up the wrong tree? Redox Biol. 2020; 29:101394. 
https://doi.org/10.1016/j.redox.2019.101394.

Kaufmann T, Strasser A, Jost PJ. Fas death receptor signalling: roles of Bid and XIAP. Cell Death Differ. 2012; 19(1):42-50. https://doi.org/10.1038/cdd.2011.121.

Kavurma MM, Khachigian LM. Signaling and transcriptional control of Fas ligand gene expression. Cell Death Differ. 2003; 10(1):36-44. https://doi.org/ 10.1038/sj.cdd.4401179.

Kumari $\mathrm{H}$, Huang WH, Chan MWY. Review on the Role of Epigenetic Modifications in Doxorubicin-Induced Cardiotoxicity. Front Cardiovasc Med. 2020; 7:56. https://doi.org/10.3389/fcvm.2020.00056.

Laptenko O, Prives C. Transcriptional regulation by p53: one protein, many possibilities. Cell Death Differ. 2006; 13(6):951-61. https://doi.org/ 10.1038/sj.cdd.4401916.

Li M, Hou T, Gao T, Lu X, Yang Q, Zhu Q, et al. p53 cooperates with SIRT6 to regulate cardiolipin de novo biosynthesis. Cell Death Dis. 2018; 9(10):941. https://doi.org/10.1038/s41419-018-0984-0.

Li RL, Wu SS, Wu Y, Wang XX, Chen HY, Xin JJ, et al. Irisin alleviates pressure overload-induced cardiac hypertrophy by inducing protective autophagy via mTOR-independent activation of the AMPK-ULK1 pathway. J Mol Cell Cardiol. 2018; 121:242-55. https://doi.org/10.1016/j.yjmcc.2018.07.250.

Li J, Wang PY, Long NA, Zhuang J, Springer DA, Zou J, et al. p53 prevents doxorubicin cardiotoxicity independently of its prototypical tumor suppressor activities. Proc Natl Acad Sci U S A. 2019; 116(39):19626-34. https://doi.org/10.1073/pnas.1904979116.

Liu X, Fan L, Lu C, Yin S, Hu H. Functional Role of p53 in the Regulation of Chemical-Induced Oxidative Stress. Oxid Med Cell Longev. 2020; 2020:6039769. https://doi.org/10.1155/2020/6039769.

Men H, Cai H, Cheng Q, Zhou W, Wang X, Huang S, et al. The regulatory roles of p53 in cardiovascular health and disease. Cell Mol Life Sci. 2020:1-18. https://doi.org/10.1007/s00018-020-03694-6

McSweeney KM, Bozza WP, Alterovitz WL, Zhang B. Transcriptomic profiling reveals p53 as a key regulator of doxorubicin-induced cardiotoxicity. Cell Death Discov. 2019; 5:102. https://doi.org/10.1038/s41420-019-0182-6.

Prathumsap N, Shinlapawittayatorn K, Chattipakorn SC, Chattipakorn N. Effects of doxorubicin on the heart: From molecular mechanisms to intervention strategies. Eur J Pharmacol. 2020; 866:172818. https://doi.org/10.1016/j.ejphar.2019.172818.

Poulose N, Raju R. Sirtuin regulation in aging and injury. Biochim Biophys Acta. 2015; 1852(11): 2442-55. https://doi.org/10.1016/j.bbadis.2015.08.017.

Ran LK, Chen Y, Zhang ZZ, Tao NN, Ren JH, Zhou L, et al. SIRT6 Overexpression Potentiates Apoptosis Evasion in Hepatocellular Carcinoma via BCL2-Associated X Protein-Dependent Apoptotic Pathway. Clin 
Cancer Res. 2016; 22(13):3372-82. https://doi.org/10.1158/1078-0432.CCR-15-1638.

Saiyang X, Deng W, Qizhu T. Sirtuin 6: A potential therapeutic target for cardiovascular diseases. Pharmacol Res. 2020; 163:105214. https://doi.org/10.1016/j.phrs.2020.105214.

Shi T, Dansen TB. Reactive Oxygen Species Induced p53 Activation: DNA Damage, Redox Signaling, or Both? Antioxid Redox Signal. 2020; 33(12):839-59. https://doi.org/10.1089/ars.2020.8074.

Shizukuda Y, Matoba S, Mian OY, Nguyen T, Hwang PM. Targeted disruption of p53 attenuates doxorubicin-induced cardiac toxicity in mice. Mol Cell Biochem. 2005; 273(1-2): 25-32. https://doi.org/10.1007/s11010-005-5905-8.

Stephanou A, Scarabelli TM, Brar BK, Nakanishi Y, Matsumura M, Knight RA, et al. Induction of apoptosis and Fas receptor/Fas ligand expression by ischemia/reperfusion in cardiac myocytes requires serine 727 of the STAT-1 transcription factor but not tyrosine 701. J Biol Chem. 2001; 276(30):28340-7. https://doi.org/10.1074/jbc.M101177200.

Sullivan KD, Galbraith MD, Andrysik Z, Espinosa JM. Mechanisms of transcriptional regulation by p53. Cell Death Differ. 2018; 25(1): 133-43. https://doi.org/10.1038/cdd.2017.174.

Tao H, Shi KH, Yang JJ, Huang C, Zhan HY, Li J. Histone deacetylases in cardiac fibrosis: current perspectives for therapy. Cell Signal. 2014; 26(3):521-7.https://doi.org/10.1016/j.cellsig.2013.11.037.

Tourneur L, Chiocchia G. FADD: a regulator of life and death. Trends Immunol. 2010; 31(7), 260-9. https://doi.org/10.1016/j.it.2010.05.005.

Ueno M, Kakinuma Y, Yuhki K, Murakoshi N, lemitsu M, Miyauchi T, et al. Doxorubicin induces apoptosis by activation of caspase- 3 in cultured cardiomyocytes in vitro and rat cardiac ventricles in vivo. $J$ Pharmacol Sci. 2006; 101(2):151-8. https://doi.org/10.1254/jphs.fp0050980.

Wang X, Wang XL, Chen HL, Wu D, Chen JX, Wang XX, et al. Ghrelin inhibits doxorubicin cardiotoxicity by inhibiting excessive autophagy through AMPK and p38-MAPK. Biochem Pharmacol. 2014; 88(3): 334-50. https://doi.org/10.1016/j.bcp.2014.01.040.

Wang XX, Wang XL, Tong MM, Gan L, Chen H, Wu SS, et al. SIRT 6 protects cardiomyocytes against ischemia/reperfusion injury by augmenting FoxO3a-dependent antioxidant defense mechanisms. Basic Res Cardiol. 2016; 111(2): 13. https://doi.org/ 10.1007/s00395-016-0531-z.

Yamada A, Arakaki R, Saito M, Kudo Y, Ishimaru N. Dual Role of Fas/FasL-Mediated Signal in Peripheral Immune Tolerance. Front Immunol. 2017; 8:403. https://doi.org/10.3389/fimmu.2017.00403.

Yang M, Zhang Y, Ren J. Acetylation in cardiovascular diseases: Molecular mechanisms and clinical implications. Biochim Biophys Acta Mol Basis Dis. 2020; 1866(10):165836.

https://doi.org/10.1016/j.bbadis.2020.165836. 
Zhang YW, Shi J, Li YJ, Wei L. Cardiomyocyte death in doxorubicin-induced cardiotoxicity. Arch Immunol Ther Exp. 2009; 57(6):435-45. https://doi.org/10.1007/s00005-009-0051-8.

Zhang D, Lin J, Han J. Receptor-interacting protein (RIP) kinase family. Cell Mol Immunol. 2010. 7(4):2439. https://doi.org/10.1038/cmi.2010.10.

Zhang T, Zhang Y, Cui M, Jin L, Wang Y, Lv F, et al. CaMKII is a RIP3 substrate mediating ischemia- and oxidative stress-induced myocardial necroptosis. Nat Med. 2016; 22(2):175-82.

https://doi.org/10.1038/nm.4017.

Zhu W, Soonpaa MH, Chen H, Shen W, Payne RM, Liechty EA, et al. Acute doxorubicin cardiotoxicity is associated with p53-induced inhibition of the mammalian target of rapamycin pathway. Circulation. 2009; 119:99-106. https://doi.org/ 10.1161/CIRCULATIONAHA.108.799700.

\section{Supplemental Figures}

Supplemental figures are not available in this version.

\section{Figures}




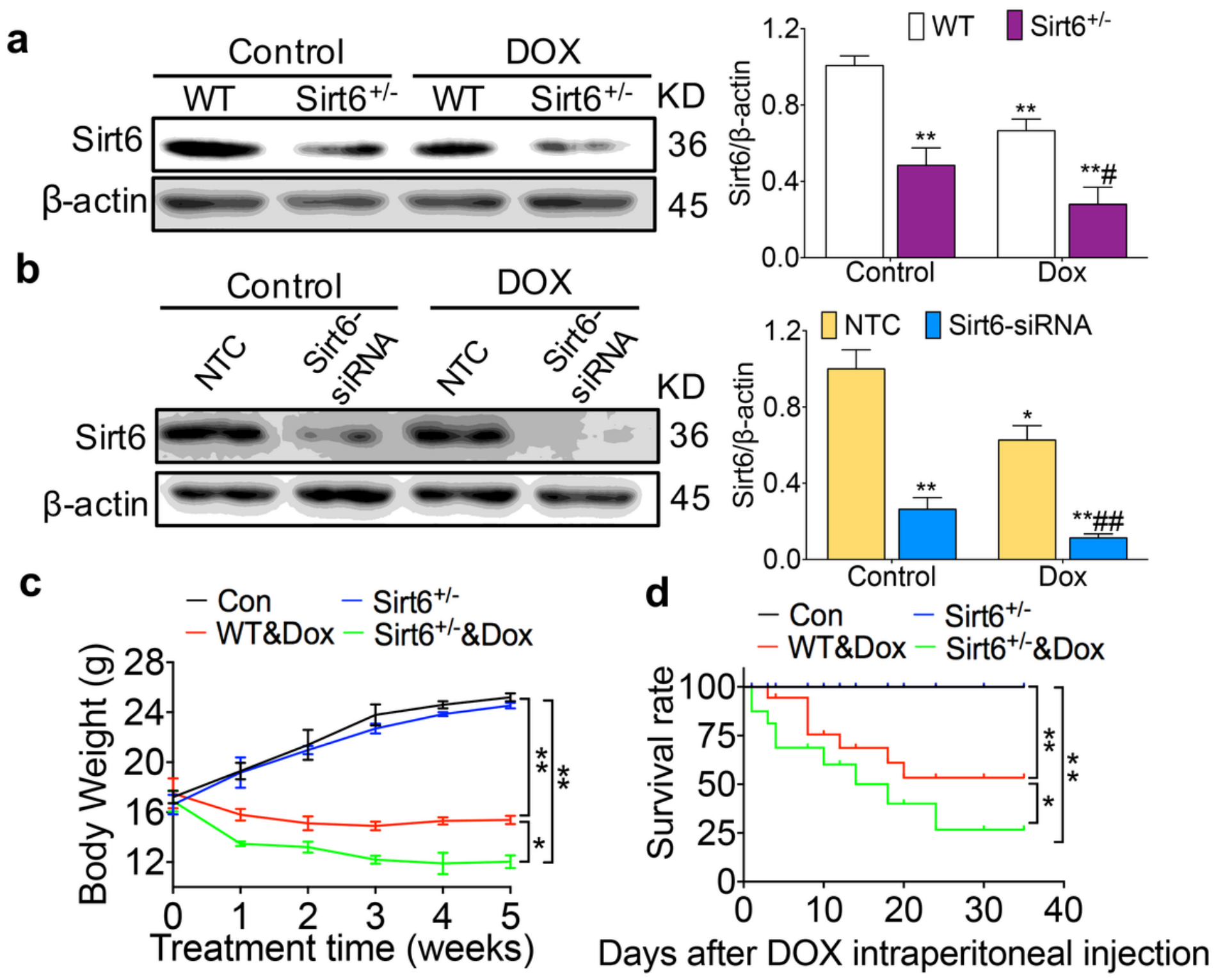

Figure 1

Sirt6 is downregulated by doxorubicin (DOX) treatment in cardiac tissues or neonatal rat cardiomyocytes (NRCMs), Sirt6 partial abolition aggregated the body weight loss and mortality in mice with DOX treatment. a Sirt6 protein level determined by western blot analysis in hearts of wild-type (WT) or Sirt6+/mice without or with DOX ( $8 \mathrm{mg} / \mathrm{kg}$ weekly) for 4 weeks, and maintenance for another one week ( $\mathrm{n}=$ 5/group). * $P<0.05$, ** $P<0.01$ vs wild-type (WT) Control mice. \# $P<0.05$ vs DOX-treated WT mice. $b$ Sirt6 protein level determined by western blot analysis in NRCMs transfected with Sirt6-siRNA or NTC (non-target control siRNA) for $48 \mathrm{~h}$, then without or with DOX $(2 \mu \mathrm{M})$ for $18 \mathrm{~h}(\mathrm{n}=5 /$ group $)$. ** $\mathrm{P}<0.01$ vs NTC, \# P $<0.05$ vs DOX-treated NTC. c Body weight alterations with DOX treatment in WT and Sirt6+/mice ( $n=10$ /group). ${ }^{\star} P<0.05$, ${ }^{\star} \mathrm{P}<0.01$. $d$ Cumulative survival of wild-type or Sirt6+/- mice without or with DOX ( $8 \mathrm{mg} / \mathrm{kg}$ weekly) for 4 weeks and maintenance for another one week, by Kaplan-Meier analysis ( $n=10$ /group). ${ }^{\star} P<0.05, * \star P<0.01$. In Fig. a-c all results were analyzed by one-way ANOVA, and values represent the mean \pm SEM. 


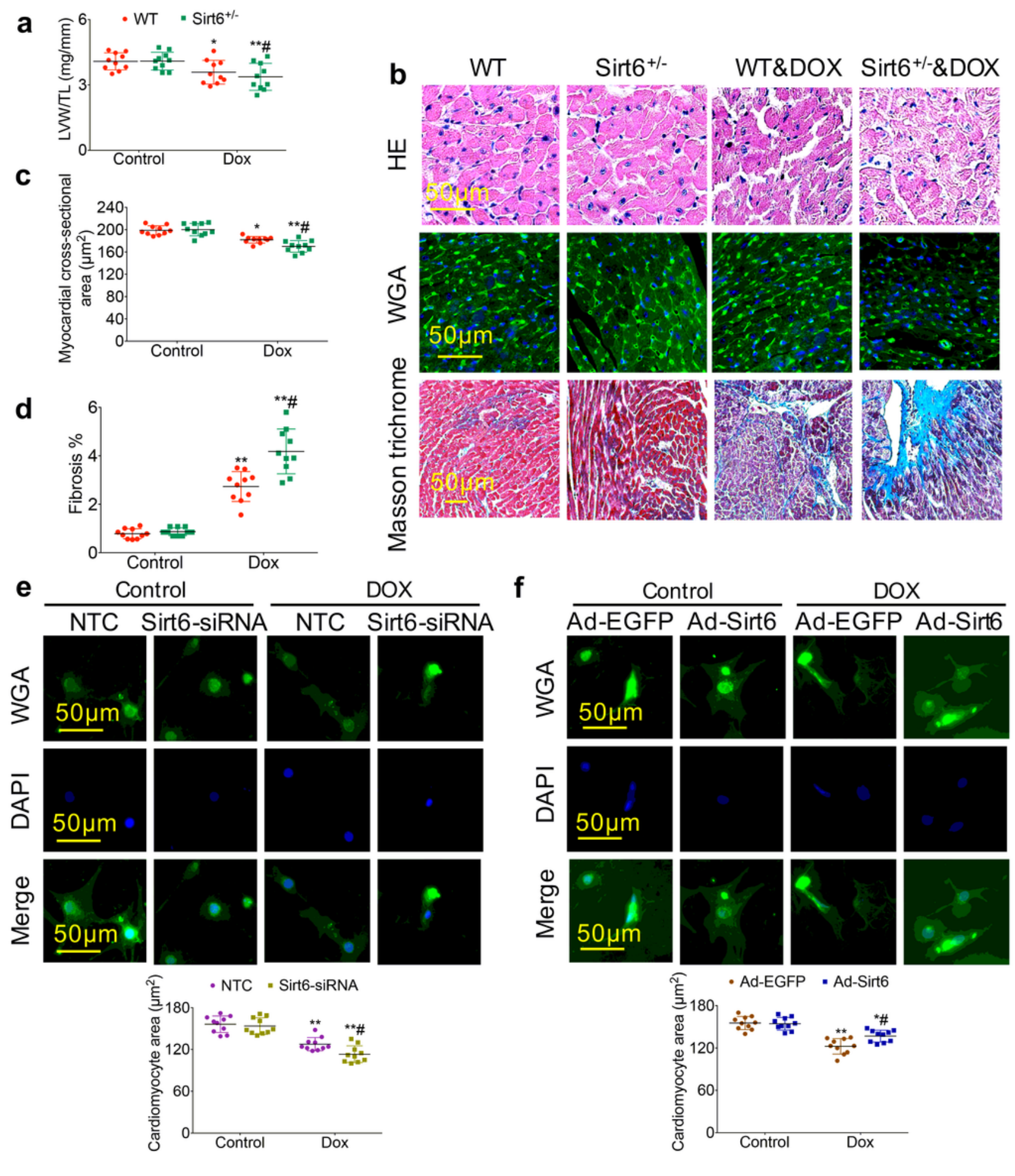

Figure 2

Sirt6 deficiency aggravates the ratio of left ventricular weight to tibal length (LVW/TL囚changes, cardiac atrophy and fibrosis following DOX injection in mice, as well as cardiac atrophy in cultured NRCMs exposed to DOX. a Changes in LVW/TL ratio in WT or Sirt6+/- mice without or with DOX (8 $\mathrm{mg} / \mathrm{kg}$ weekly) injection for 4 weeks and maintenance for another one week ( $n=10$ /group). b Representative images of hematoxylin and eosin (HE)-, wheat germ agglutinin (WGA)- and Masson trichrome (blue represents 
collagen)-stained heart sections. Original magnifications, $\times 200, \times 400$ and $\times 100$ respectively; all scale bars $=50 \mu \mathrm{m} . \mathrm{c}, \mathrm{d}$ Quantitative measurement of cardiomyocyte cross-sectional area with random 100200 cardiomyocytes ( $n=10$ /group); the percentage of fibrosis with normalizing blue Masson's trichrome-stained area to LV area in five randomly selected fields per section ( $n=10 /$ group). e, $f$ Representative images of WGA-Alexa Fluor 488-stained NRCMs. NRCMs transfected with Sirt6 siRNA or NTC siRNA for knockdown for $48 \mathrm{~h}$ or infected with Ad-EGFP or Ad-Sirt6 adenovirus for overexpression for $48 \mathrm{~h}$ followed by treatment without or with DOX $(2 \mu \mathrm{M})$ for $18 \mathrm{~h}(\mathrm{n}=10 / \mathrm{group})$, then cell area was measured with WGA-Alexa Fluor 488 staining: the cell membrane is green and nuclei is blue. Scale bars $=$ $50 \mu \mathrm{m}$. Original magnification, $\times 400$. Quantitative analysis of NRCM size with measurements of $\geq 50$ NRCMs per group. All data were analyzed by one-way ANOVA, and values represent the mean \pm SEM. In Fig. a, $c, d{ }^{* * P}<0.01$ vs the WT control, \#\#P<0.01 vs DOX-treated WT mice. In Fig. ${ }^{*} \mathrm{P}<0.05, * \star P<0.01$ vs NTC, \#P< 0.05 vs DOX-treated NTC. In Fig. $f * P<0.05$, ** $P<0.01$ vs Ad-EGFP, \#P<0.05 vs DOXtreated Ad-EGFP. 


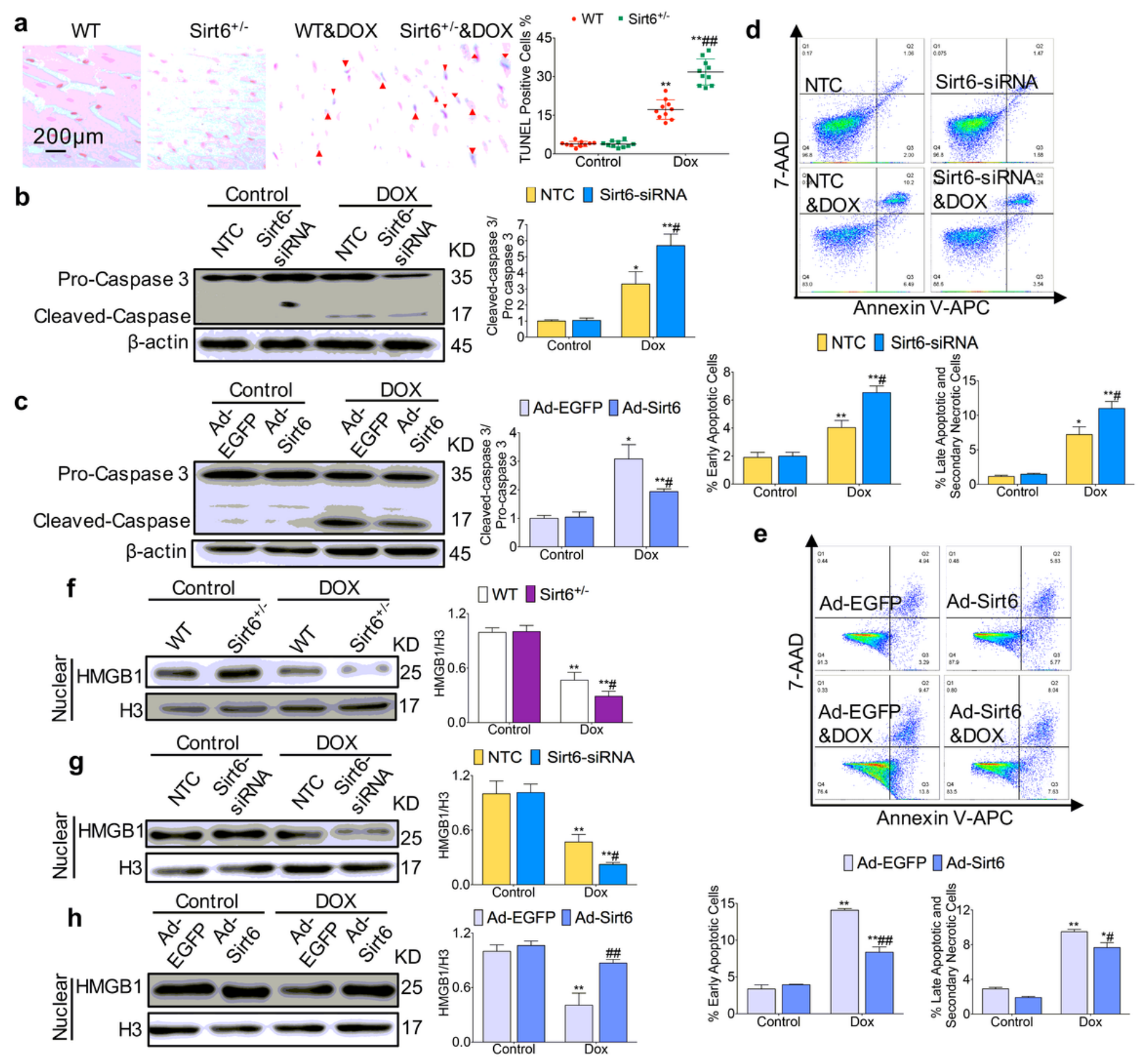

\section{Figure 3}

The effect of knockdown/overexpression of Sirt6 on DOX-induced apoptosis and necrosis in mouse hearts and NRCMs. a Representative images of TUNEL-stained sections in hearts of WT or Sirt6+/- mice without or with DOX (8 mg/kg weekly) for 4 weeks and maintenance for another one week ( $\mathrm{n}=$ 10/group). Red arrows indicate some blue-stained TUNEL-positive nuclei. Scale bars $=50 \mu \mathrm{m}$. Original magnification, $\times 400$. Quantitative analysis of TUNEL-positive cells as a percentage of positive nuclei number to total nuclei number in all groups ( $n=10$ /group). ${ }^{*} P<0.01$ vs the WT Control, \#\#P<0.01 vs DOX-treated WT. b, c Pro- and cleaved caspase-3 protein levels measured by western blot assay ( $\mathrm{n}=$ 5/group) in NRCMs exposed to DOX ( $2 \mu \mathrm{M})$ for $18 \mathrm{~h}$ after transfection with NTC or Sirt6 siRNA for $48 \mathrm{~h}$ or

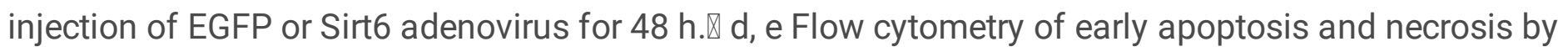


staining with Annexin V-APC and 7-ADD. NRCMs were transfected with NTC or Sirt6-siRNA or infected with EGFP or Sirt6 adenovirus, then treated with $2 \mu \mathrm{M}$ Dox for $18 \mathrm{~h}$. Early apoptosis is annexin $\mathrm{V}$ and 7ADD-positive (lower left quadrant). Late apoptotic and secondary necrotic cells are both APC and 7$A A D$-positive (upper right quadrant) and $A A D$ - positive (upper left quadrant). The percentage of apoptotic and necrotic cells was quantified $(n=5)$. f-h HMGB1 (nuclear part) protein level in all groups by immunoblotting $(n=5)$. All data were analyzed by one-way ANOVA, and values represent the mean \pm SEM. In Fig. $a, f * P<0.05,{ }^{*} P<<0.01$ vs the WT Control, \#P<0.05, \#\#P<0.01 vs DOX-treated WT. In Fig. b, $d, g * P<0.05,{ }^{*} P<0.01$ vs NTC, $\# P<0.05$ vs DOX-treated NTC. In Fig. $c, e, h * P<0.05, * * P<0.01$ vs Ad-EGFP, \#P< 0.05 vs DOX-treated Ad-EGFP.

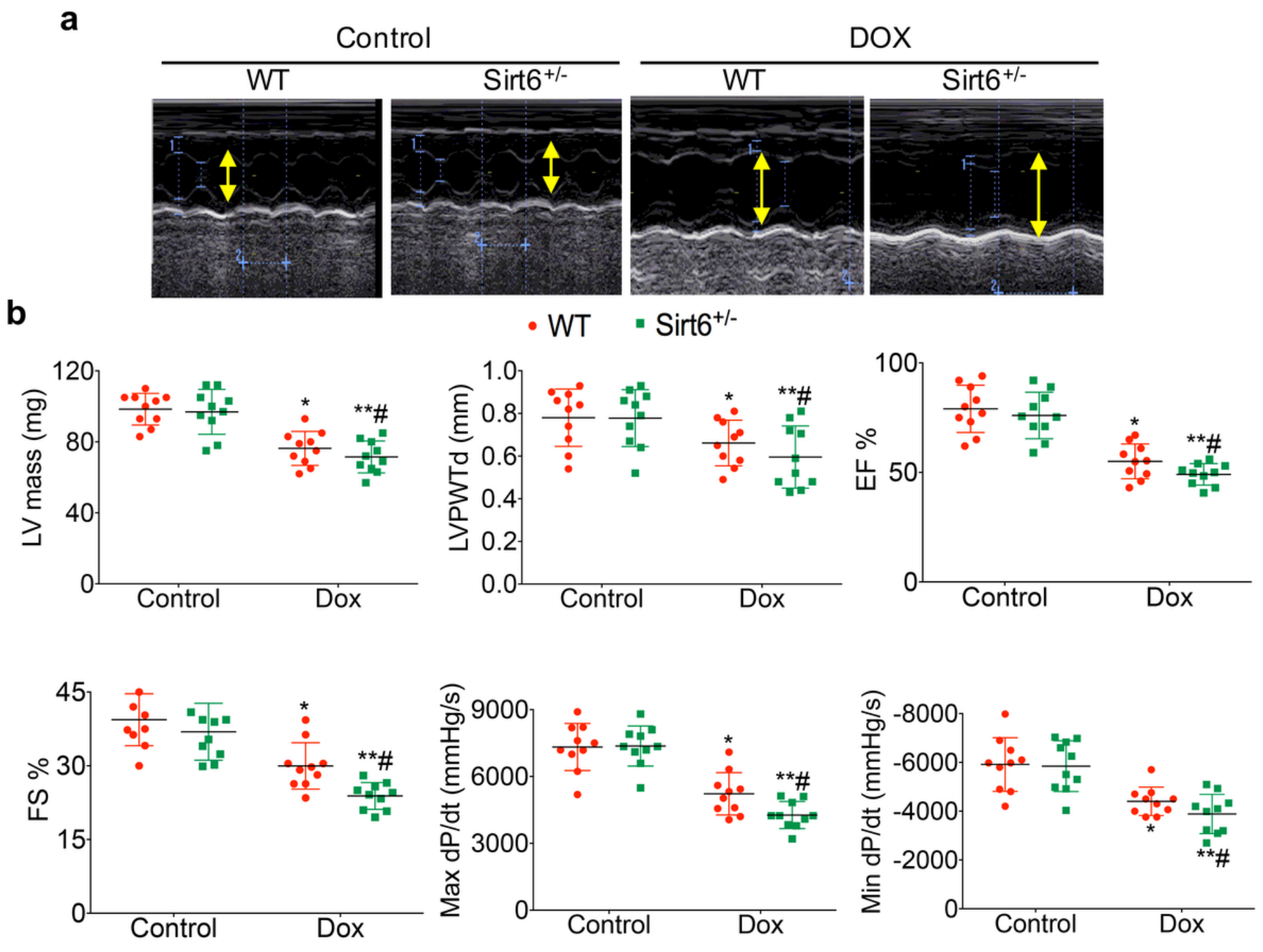

Figure 4

Loss of Sirt6 accelerates left ventricular dysfunction after DOX treatment. a Representative echocardiographs and echocardiographic parameters of WT, Sirt6+/-, and WT or Sirt6+/- mice after DOX ( $8 \mathrm{mg} / \mathrm{kg}$ weekly) for 4 weeks and maintenance for another one week $(n=10 /$ group). Yellow arrow 
indicates width of the LV chamber. b Values for LV mass, LVPWTd, EF\%, FS\%, Max dP/dt and Min dP/dt for mice ( $n=10$ /group). All data were analyzed by one-way ANOVA. ${ }^{*} P<0.05,{ }^{*} P<0.01$ vs WT control; $\# p<0.05$ vs DOX-treated WT; LVPWTd, left ventricle (LV) posterior wall thickness at end-diastole; EF\%, ejection fraction \%; FS\%, LV fractional shortening \%; Max dP/dt, maximal value of the first derivative of LV pressure; Min $\mathrm{dP} / \mathrm{dt}$, minimal value of the first derivative of LV pressure.
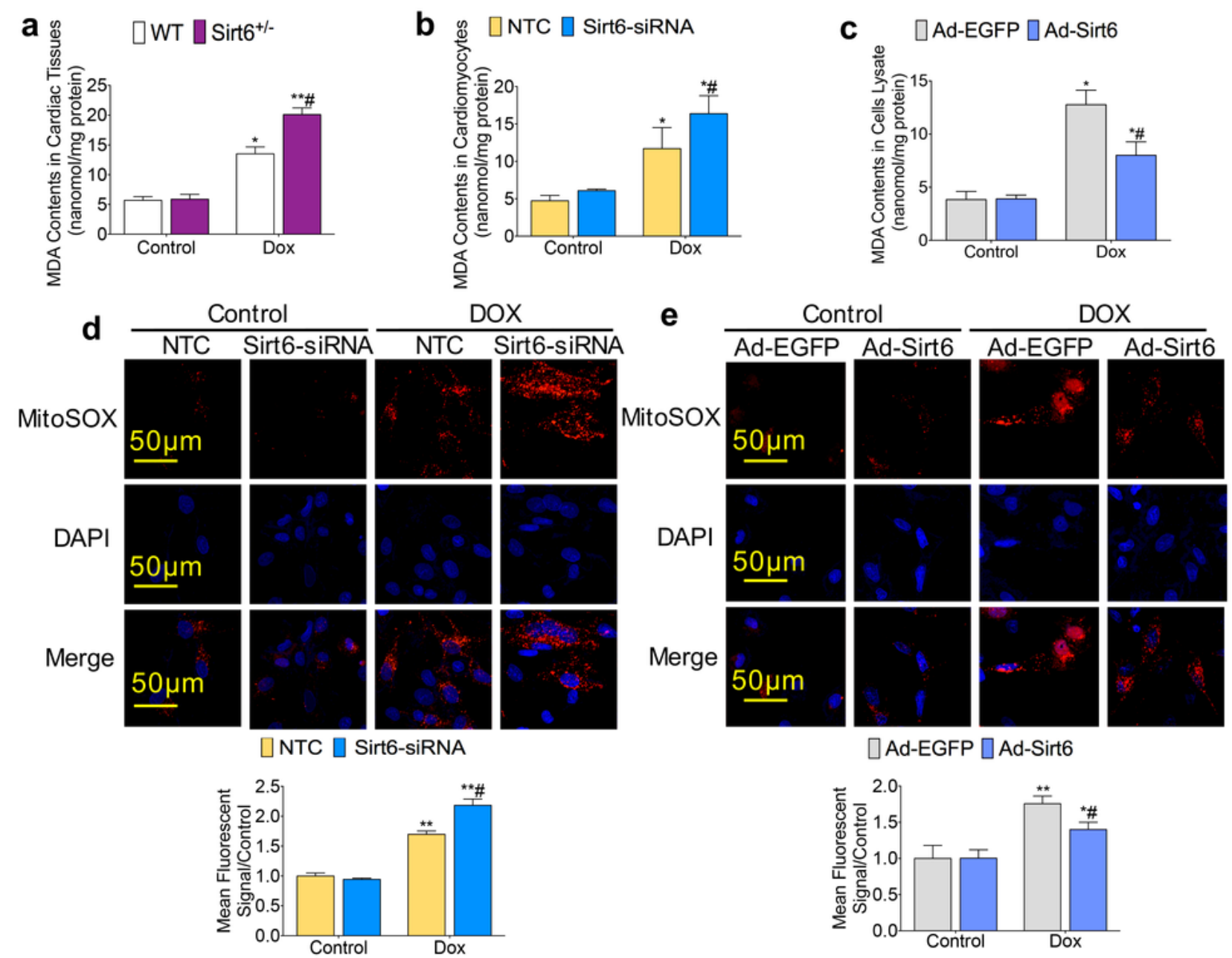

૧Ad-EGFP $\square$ Ad-Sirt6
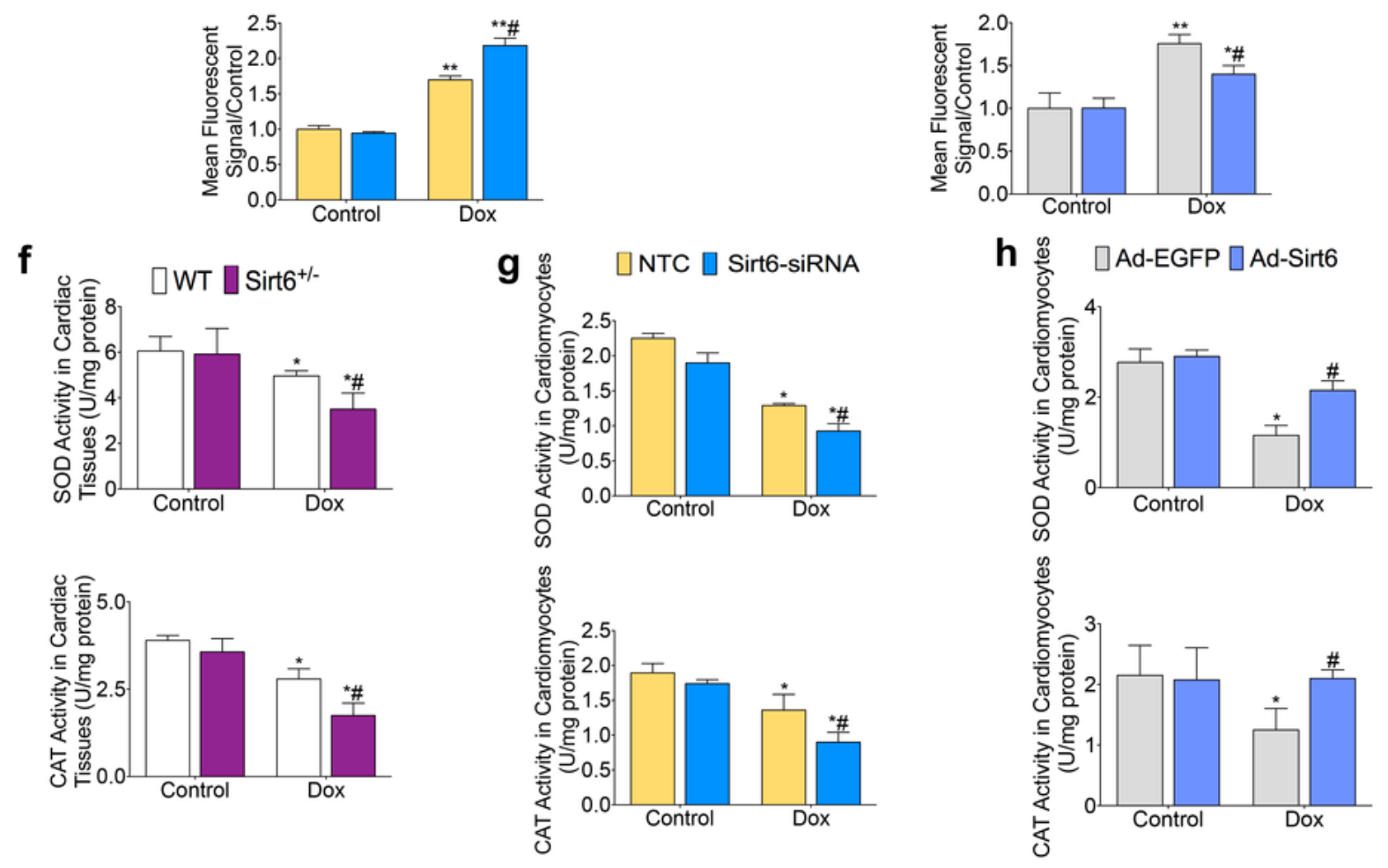

Figure 5 
Effect of Sirt6 deficiency/overexpression on DOX-induced mitochondria oxidative stress in myocardial tissues or NRCMs. a Malondialdehyde (MDA) content in mouse cardiac tissue homogenates of WT or Sirt6+/- mice without or with DOX (8mg/kg weekly) for 4 weeks and maintenance for another one week (n $=5 /$ group). $b, c$ MDA content in cultural supernatants of NTC and Sirt6 siRNA NRCMs or Ad-EGFP and Ad-Sirt6-infected NRCMs, without or with $2 \mu$ M DOX for $18 \mathrm{~h}$ ( $n=5$ /group). d, e Representative MitoSOXred staining images and quantitative fluorescence intensity results of reactive oxygen species levels in NTC and Sirt6 siRNA NRCMs or Ad-EGFP and Ad-Sirt6-infected NRCMs, without or with $2 \mu$ M DOX for 18 $h(n=5 /$ group). Scale bar $=50 \mu \mathrm{m}$. $f$ Activity levels of superoxide dismutase (SOD2) and catalase (CAT) in mouse cardiac tissue homogenates of WT or Sirt6+/- mice without or with DOX ( $8 \mathrm{mg} / \mathrm{kg}$ weekly) injection for 4 weeks and maintenance for another one week ( $n=5 /$ group). g-h Activity levels of SOD2 and CAT in cultural supernatants of NTC and Sirt6 siRNA NRCMs or Ad-EGFP and Ad-Sirt6-infected NRCMs, without or with $2 \mu \mathrm{M} \mathrm{DOX}$ treatment for $18 \mathrm{~h}$ ( $\mathrm{n}=5$ /group). All data were analyzed by one-way ANOVA, and values represent the mean \pm SEM. In Fig. a, $f * P<0.05$, $* * P<0.01$ vs the WT Control, \#P< 0.05 vs DOX-treated WT. In Fig. $b, d, g * P<0.05$, ${ }^{*} P<0.01$ vs NTC, \#P<0.05 vs DOX-treated NTC. In Fig. $c, e, h * P<0.05$ vs Ad-EGFP, \#P $<0.05$ vs DOX-treated Ad-EGFP. 

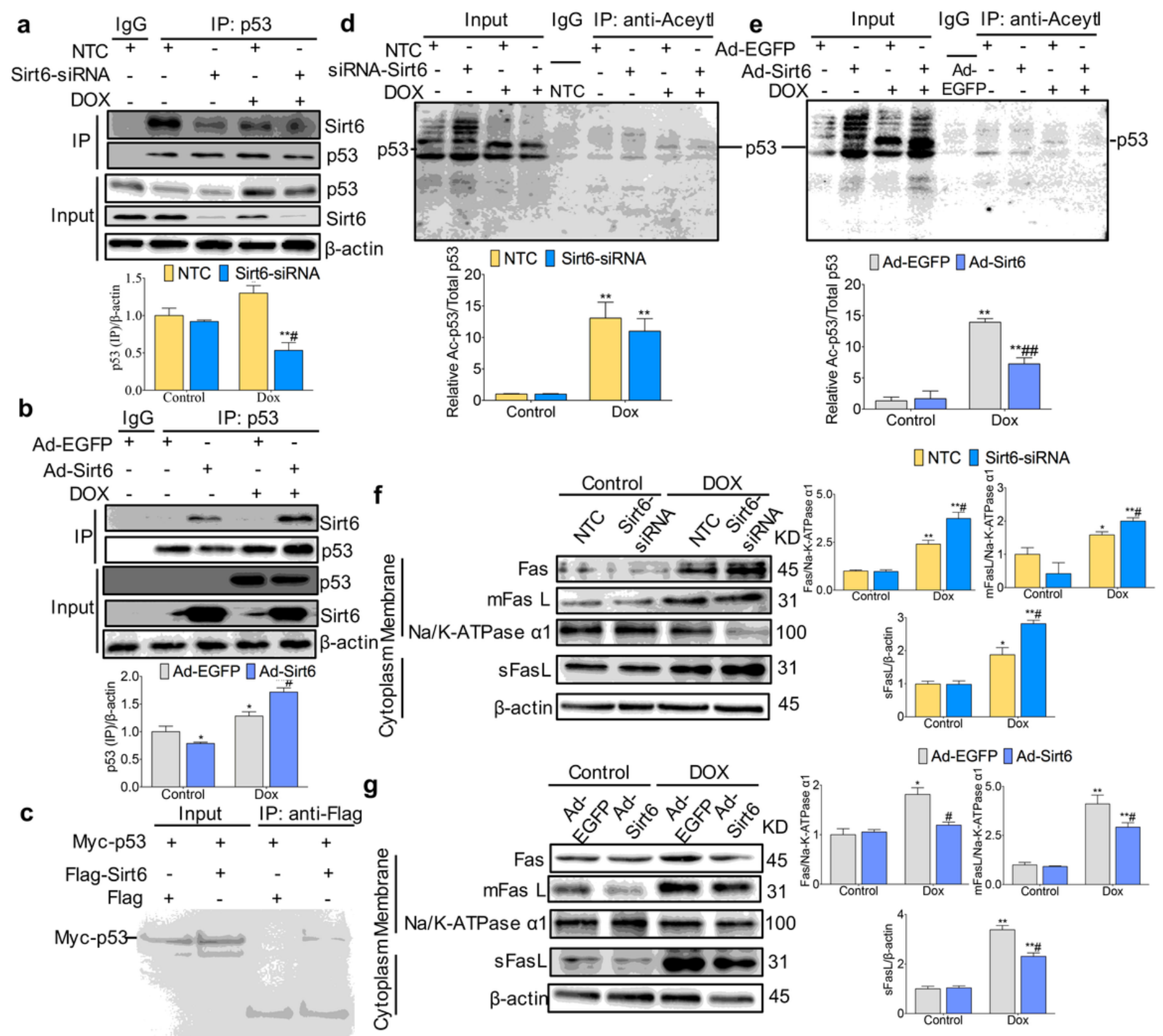

\section{Figure 6}

Effect of Sirt6 deficiency/overexpression on DOX-insulted cardiomyocyte death is partially mediated by a p53-Fas dependent pathway in NRCMs. a, b NRCMs were transfected with Sirt6-siRNA or NTC or infected with Ad-Sirt6 or Ad-EGFP without or with DOX $(2 \mu \mathrm{M})$ for $18 \mathrm{~h}$. Cell lysates were extracted for Co-IP with IgG or anti-p53 antibody and probed with the indicated antibodies. Densitometry of relative p53 protein content to $\beta$-actin $(n=3)$. c HEK293T cells transfected with Myc-tagged p53 and with Flag-tagged Sirt6 or Flag for $24 \mathrm{~h}$. Cell lysates were incubated with IgG or anti-Flag antibody and the input and immune complexes were immunoblotted with anti-Myc antibody by western blot analysis. $d$, e NRCMs were transfected with Sirt6-siRNA or NTC or infected with Ad-Sirt6 or Ad-EGFP without or with DOX $(2 \mu \mathrm{M})$ for $18 \mathrm{~h}$. Cell lysates were extracted for Co-IP with IgG or anti-p53 antibody and probed with the acylation antibody. Representative images are from three independent experiments. f, g NRCMs were transfected 
with Sirt6-siRNA or NTC or infected with Ad-Sirt6 or Ad-EGFP without or with DOX $(2 \mu \mathrm{M})$ for $18 \mathrm{~h}$. Membrane and cytoplasm proteins were extracted and analyzed by western blot analysis. Expression of Fas, mFasL (membrane FasL), Na/K-ATPase a1, sFasL (soluble FasL) and $\beta$-actin were determined by using specific antibodies ( $n=5 /$ group). All data were analyzed by one-way ANOVA. Values represent the mean \pm SEM. In Fig. $a, f * p<0.05$, ${ }^{*} P<0.01$ vs NTC, \#P< 0.05 vs DOX-treated NTC. In Fig. $B, G * P<0.05$, $* * P<0.01$ vs Ad-EGFP, \#P $<0.05$ vs DOX-treated Ad-EGFP.

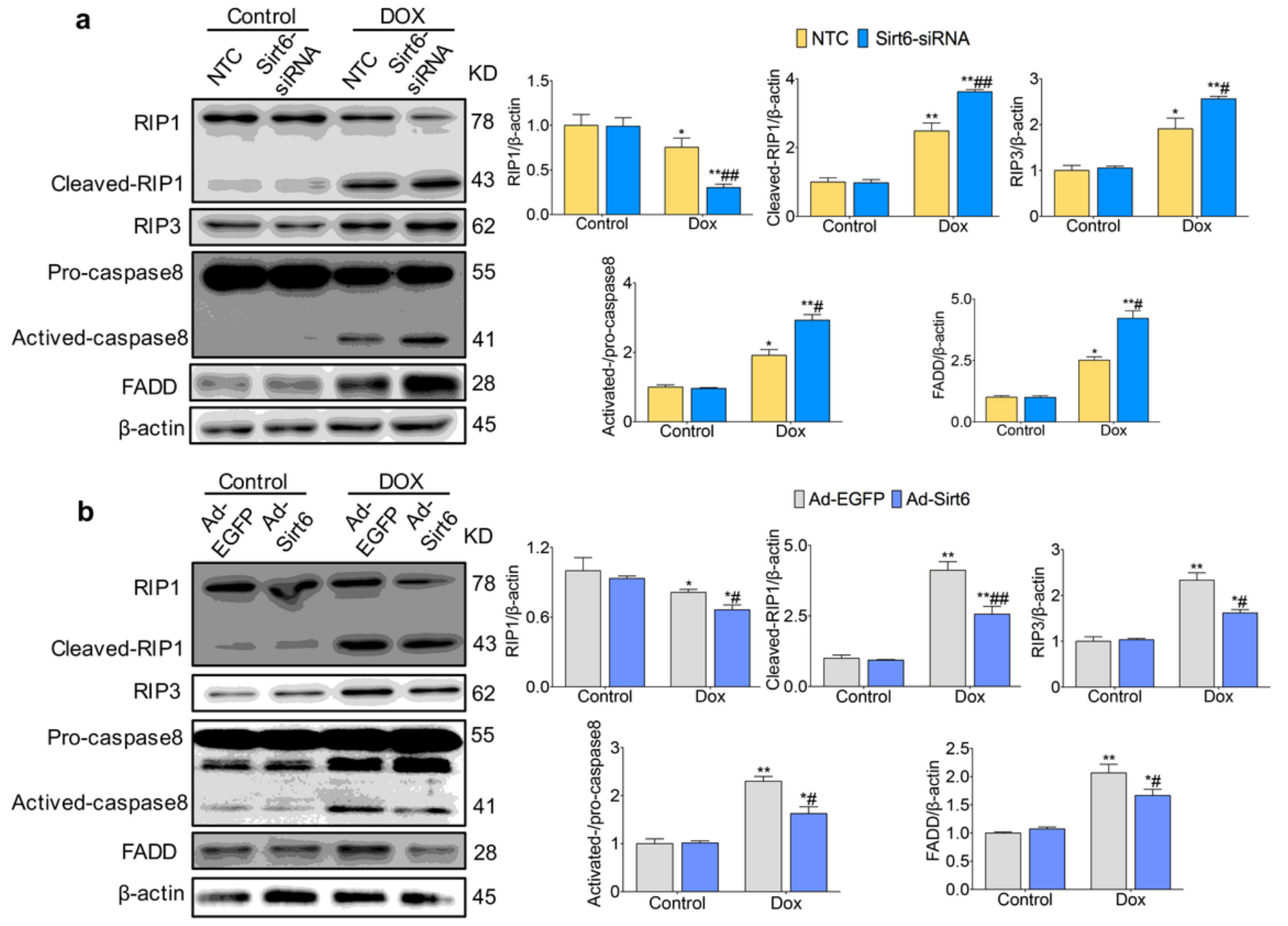

Figure 7

Effect of Sirt6 deficiency/overexpression on DOX-insulted cardiomyocyte apoptosis is caspase-8dependent and necrosis is RIP1-independent in NRCMs. a, b NRCMs were transfected with Sirt6-siRNA or NTC or infected with Ad-Sirt6 or Ad-EGFP without or with DOX $(2 \mu \mathrm{M})$. Immunoblotting of cell lysates for expression of RIP1, RIP3, Caspase8, FADD and $\beta$-actin with specific antibodies ( $n=5 /$ group). All data were analyzed by one-way ANOVA. Values represent the mean \pm SEM. In Fig.a $* P<0.05$, $* \star P<0.01$ vs NTC, \#P<0.05, \#\#P<0.01 vs DOX-treated NTC. In Fig. $b * P<0.05$, ** $P<0.01$ vs Ad-EGFP, \#P<0.05 vs DOX-treated Ad-EGFP. 
a

$\square$ NTC $\square$ NTC\&DOX

\Sirt6-siRNA \ Sirt6-siRNA\&DOX

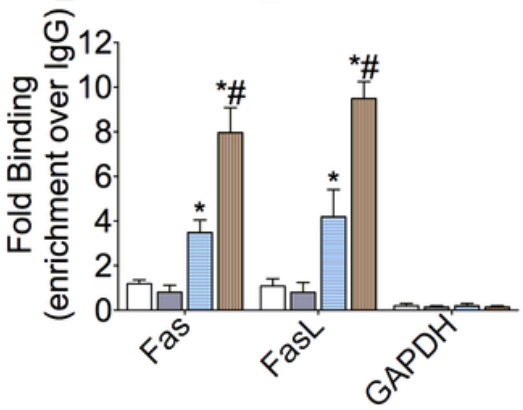

b

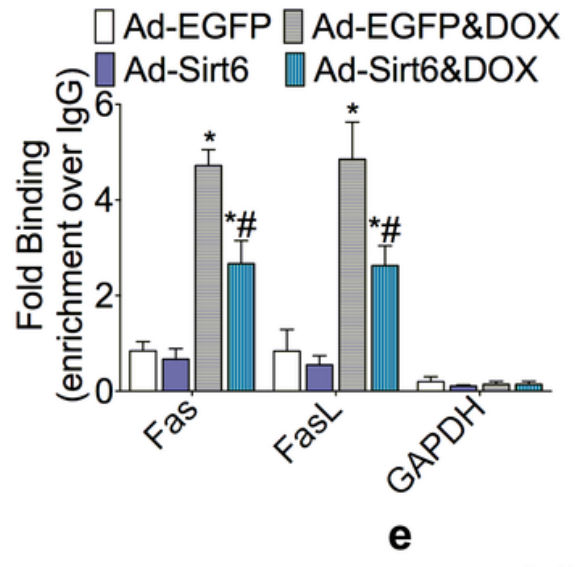

C

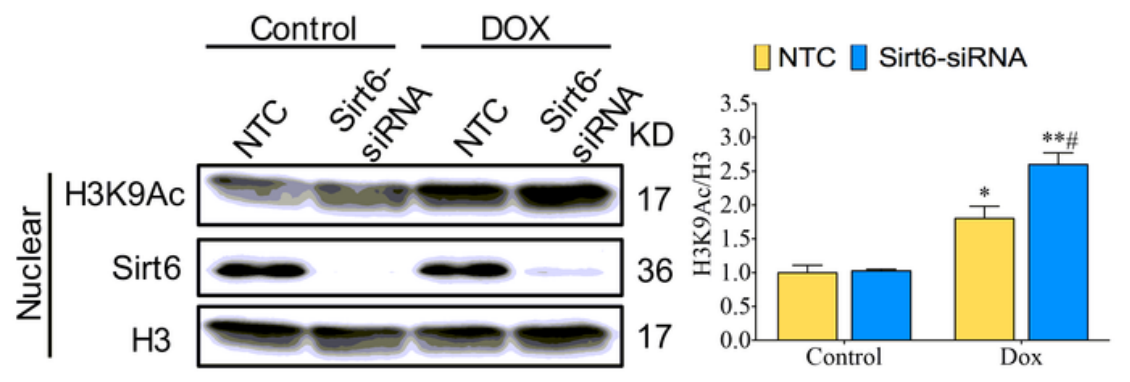

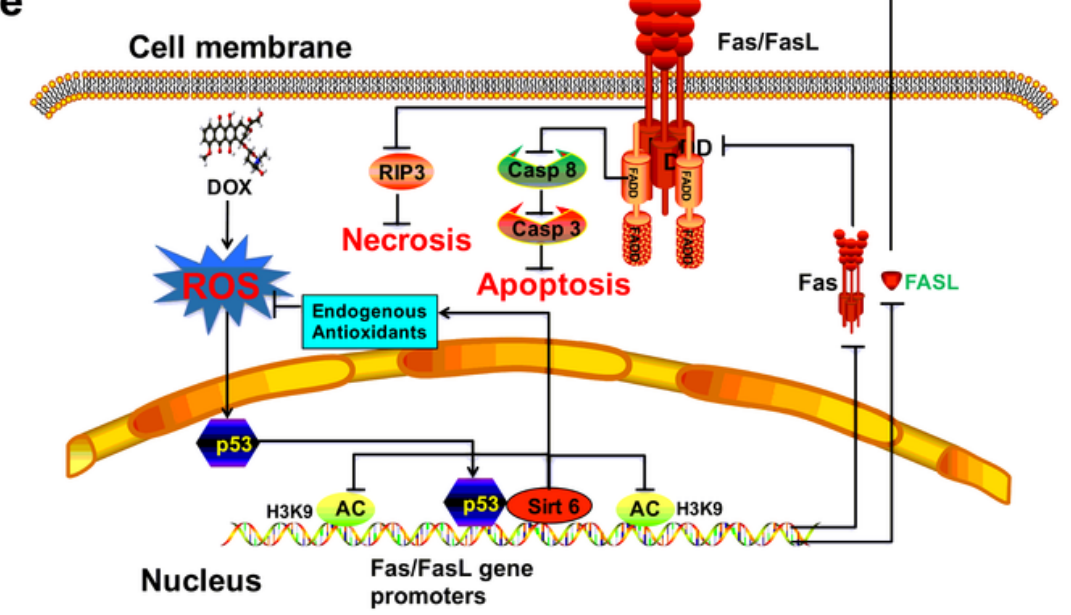

Figure 8

8 Endogenous Sirt6 interacts with p53 and plays an important role in mediating the expression of its target gene products, FASL and FAS, transcriptionally in NRCMs. a, b Chromatin immunoprecipitation (ChIP) analysis to detect p53 binding at the promoter of Fas or FasL, with p53-specific antibody and immunoglobulin G (IgG) control, in NRCMs transfected with Sirt6-siRNA or NTC or infected with Sirt6 or control adenovirus for $48 \mathrm{~h}$ with or without DOX $(2 \mu \mathrm{M})$ for $18 \mathrm{~h}$. PCR analysis involved p53 sites in Fas or FasL promoter; IgG-ChIP was a negative control; the summarized data is relative to the IgG control antibody background ( $n=5$ /group). c, d NRCMs were transfected with Sirt6-siRNA or NTC or infected with Sirt6 or control adenovirus with or without DOX $(2 \mu \mathrm{M})$ for $18 \mathrm{~h}$. Nuclear protein was analyzed by immunoblotting. H3K9Ac level was determined by western blot analysis. Densitometry analysis of relative protein content to $\mathrm{H} 3$ ( $n=3$ /group). e The schematic diagram summarizing the signaling 
pathways involved in Sirt6 regulating DOX-induced cardiotoxicity. DOX exposure induces a significant increase in oxidative stress, apoptosis and necrosis, which does harm to cardiomyocytes and contributes to DOX-induced cardiotoxicity. Sirt6 protects against DOX-induced cardiac injury by upregulating endogenous antioxidants and interacting with p53 as a co-repressor. Sirt6 is recruited to the gene promoters of death receptor, Fas, and its ligand, FasL, to suppress p53 transcription activity by reducing histone acetylation. Sirt6 inhibits death receptor signaling to further suppress Fas-FADD-caspase-8 apoptotic and Fas-RIP3 necrotic pathways. All data were analyzed by one-way ANOVA. Values represent the mean \pm SEM. In Fig. a, $c,{ }^{*} P<0.05,{ }^{*} \mathrm{P}<0.01$ vs NTC, \#P<0.05 vs DOX-treated NTC. In Fig. $b, d * P<$ $0.05, * * P<0.01$ vs Ad-EGFP, \#P<0.05 vs DOX-treated Ad-EGFP.

\section{Supplementary Files}

This is a list of supplementary files associated with this preprint. Click to download.

- OnlineSupplementalMaterial.docx 